\title{
Leveraging Transmedia storytelling to engage tourists in the understanding of the destination's local heritage
}

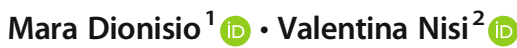

Received: 10 March 2020 /Revised: 6 February 2021 / Accepted: 14 April 2021 /

Published online: 7 June 2021

(C) The Author(s) 2021

\begin{abstract}
Transmedia Stories are becoming an increasingly important technique for the tourism industry. They are successful tools to engage, inspire and gather audiences online and offline. In this article, we describe the design, implementation and evaluation of Fragments of Laura, a bespoke Transmedia Storytelling (TS) experience designed to involve visitors in developing knowledge and awareness about the cultural and natural heritage of Madeira Island. Fragments of Laura $(\mathrm{FoL})$ is composed of two interconnected components: a Location-Aware Multimedia Story, and a Hypermedia Platform populated with locally collected testimonies and interviews. Results from the extensive evaluation of Fragments of Laura, highlights the potential of interactive multimedia, TS in particular, in engaging tourists with the destination values and community. Our contribution is twofold, on one hand we extend on the state of the art of multimedia interactive storytelling, with the description of the Fragments of Laura TS artifact. On the other hand, results from FoL evaluation highlight how the artifact impacts on the tourism experience its implications for the design of future tourism driven TS experiences.
\end{abstract}

Keywords Multimedia $\cdot$ Hypermedia $\cdot$ Transmedia storytelling $\cdot$ Narrative persuasion · Sustainable tourism $\cdot$ Location-aware multimedia stories

\section{Introduction}

Tourism is now an activity within reach of millions and this has enabled the travel industry to flourish globally. In particular, islands are unique places with a natural attraction for

Mara Dionisio

msgdionisio@gmail.com

1 ITI/LARSyS, Instituto Superior Tecnico, Faculdade de Ciências e Tecnologia, Universidade Nova, Lisbon, Portugal

2 ITI /LARSyS, Instituto Superior Tecnico, U. Lisbon, Computer Science and Engineering, Lisbon, Portugal 
tourists. For small islands, tourism represents an economic catalyst to enlarge its economy and overcome the disadvantages of smallness [15]. However, the allure of many islands (such as the Macaronesian islands for example) is dependent on a blend of unique land formations, flora and fauna, ocean and coastal resources. On the other hand the tourism captivated by this charm often contributes to the island's environmental degradation (pollution, erosion, etc.). Furthermore, to keep the small island's appeal alive and thriving, the growth of the travel and tourism sectors must continue with the investment and development taking place in an open and sustainable manner, motivating the protection of nature, habitats, and biodiversity [9, 76].

Transmedia Storytelling (TS) can play an essential role in raising awareness towards sustainable tourism practices due to its immersive and participatory nature. TS can be described as transferring and developing stories that can be spread through various media platforms. According to the definition of Henry Jenkins, TS is "a process where integral elements of a fiction get dispersed systematically across multiple delivery channels to create a unified and coordinated entertainment experience" [33]. Stories can be powerful forces that can help us understand the world and how to live our lives [23]. Narrative persuasion and character involvement are known constructs contributing to the understanding of the power of storytelling [27]. By designing stories that emotionally touch their audiences we create positive social change, educate and engage audiences on a wide range of issues, and ultimately even persuade them to take action [28].

Moreover, the transmedia aspect of storytelling can entertain through participation, engagement, interaction or collaboration of the audience. Consequently, TS takes advantage of the rapid convergence of media and consumers to participate in rich virtual (and physical) environments that have been shown to foster real emotional engagement. We explore the potential of this approach applied to the tourism industry, where TS can enhance the tourist experience at specific destinations, by applying a new level of entertainment in which emotionally charged stories and character transformations expose and inform the audiences about local heritage, establishing new points of dialogue between locals and visitors.

In order to pursue this goal, we designed and produced, Fragments of Laura ${ }^{1}$ (FoL), a bespoke TS experience, composed of a Location-Aware Multimedia Story (FoL LAMS) and a Hypermedia Platform (FoL HP). We position our work, between what Robert Pratten refers to as Transmedia for Change, (T4C) [60] and sustainable tourism [30], aiming at promoting the local heritage through a transmedia story experience. According to Pratten, T4C is based on the belief that stories matter and need to be told to the right people at the right time. Success in this context involves engaging audiences with positive messages that inspire and motivate better choices and provide solutions that have an impact in the world. In particular in a way that increases the tourists'respect for the natural, social and cultural environments of the destination areas that they are visiting.

The contributions of this work are two folded: 1) An Artifact Research Contribution: through the detailed description of the FoL design process and features, this work advances the state of the art in the design of TS experiences applied to tourism industry; 2) An Empirical Research Contribution: by presenting the results and discussion of an extensive evaluation of the proposed artifact, this article contributes with qualitative and quantitative findings about the potential of multimedia storytelling artifacts for tourist experience in a real-world context.

${ }^{1}$ http://fol.m-iti.org/ 
Furthermore, this work provides evidence of how multimedia storytelling and in particular TS can be used to enhance the tourist's experience creating awareness towards local values and heritage by answering the three following research questions:

- RQ1: Can FoL TS provide a fulfilling and memorable touristic experience?;

- RQ2: Did the two components of the FoL TS (FoL LAMS and FoL HP) equally supported tourists to emotionally connect with the island local values and community?

- RQ3: Did FoL TS enriched the knowledge about the local destination cultural and natural heritage in a significant way? If so, what role did the different TS components play out?

The rest of this article is structured as follows: Section 2 describes relevant related work; Section 3 describes the design of the FoL TS experience and its components. Section 4 presents the evaluation procedures and the main findings. Section 5 discusses the study's insights, outcomes and limitations. Finally, in Section 6, we present the future work and conclusion, respectively.

\section{Related work}

Tourism is often described as one of the world's largest industries. Intense and disproportionate tourism growth may, however, generate damaging environmental and social effects, like for example frictions with the local communities, as visitors may not behave accordingly to the island culture or traditional values $[40,70]$. Hence there is a crescent need to ensure that the tourism industry develops in alignment with local values [25, 76]. Many small islands greatly benefit from increased economic gain from tourism, but also experience some negative environmental, economic and social consequences, such as loss of habitat areas and resources due to development and pollution; decline in biodiversity, loss of natural and archaeological heritage in the face of rapid expansion [64]. Fortunately, many islands destinations have already started to promote policies that can assist with sustainability, for example, the empowerment of the island community and culture as a necessary part of planning $[11,26]$. Cultural pride, fostered through storytelling and memory of traditions and a sense of identity are vital for the preservation of healthy community feelings and ties [48, 49]. However, meaningful integration of local culture into the tourist experience is a difficult task to accomplish [62]. On the one hand, residents need to learn about the impact tourism is having on their community [56], while on the other, tourists need to learn about the destination's unique cultural and environmental features. We envisage TS a fruitful strategy to raise visitors' awareness and empathy towards the local heritage and sustainable practices.

\subsection{The role of technology in changing the tourism experience: Entertaining and meaningful experiences}

Today's travellers are increasingly demanding authentic, experientially oriented opportunities involving meaningful interactions with locals $[34,57,70]$. Furthermore, a large body of work has drawn attention to the impact, role, and value of digital technologies in supporting these new demands [47, 70, 73]. Applications and services designed to foster the connection between tourists and locals are flourishing. Some of these services, such as CityFlocks [6], AskLocal [1] and Loqal [39] are concerned with providing recommendations and advice based 
on locals' opinions so that tourists get unbiased, genuine answers from local experts. In response to the tourist's quest for "authenticity" [13, 55, 73] locals are turned into guides, (see projects vayable.com [69], Cool Cousin [14], Urban Buddy [32]) offering travel experiences recommendations, reservations, and discounts with local businesses and even home dining opportunities. Although these platforms may offer different services and experiences the underlying principle is the same and draw inspiration from these platforms in the sense that bring together a symbiotic relationship between locals and tourists. They give the travelers the possibility to connect with the local community and enrich their experience on-site and save resources. While on the other hand locals can also have a direct connection with new cultures and may have a direct monetary benefit from it.

Moreover, due to the continuous improvements and uptake of mobile smart devices capabilities and connectivity, today's urban spaces are augmented with layers of information and multimedia content $[5,79]$. In the past two decades, we have seen the rise of rich entertaining and educating experience provided through digital media associated with urban locations $[17,18]$. The bulk of such experiences have materialized in location-based and augmented reality games $[2,3,74,78]$, exploring the boundaries between gaming and physical space. Outcomes revealed that game design needs to be strongly anchored either in a narrative or a location perspective in order to be successful [31]. Hence other approaches combine history with character-driven gaming providing visitors with exciting scavenger and treasure hunts where (virtual) characters recount the history of the place and give background information $[8,12,68,77]$ making players experience the historical events and relive stories. There are also location-based storytelling historically inspired fictions and tours [42, 54] that aim to make tourists understand the places they visit in a more interactive way than just presenting bare facts. These have showcase that neighbourhoods enriched with locally inspired mobile multimedia stories can attract tourists off the beaten tracks into less populated areas $[50,58]$.

Such contributions showcase how location-aware mobile technologies and storytelling allow participants to immerse themselves in the destination history and culture while supporting meaningful engagement with the location. Likewise, our project takes advantage of the association of digital interactive and location-aware media with urban locations, to provide rich, entertaining, educational experiences while preserving authenticity and meaning.

\subsection{The potential of Transmedia storytelling applied to tourism}

Digital media technologies afford stories to be quickly created and shared widely. TS represents arguably a recent trend and a profound transformation in storytelling as it combines the capabilities of ubiquitous computing technologies, real-life experiences, and learnerfocused pedagogy drawn from a rich ecology of content and media [24, 29] into creating a unified entertainment experience [33]. Humans have been combining entertainment and education through storytelling to produce social change for millenia [4]. Not only humans are innate storytellers [63], but we are also innately prepared to be influenced by stories [27, 46]. Audiences tend to be less resistant to socially responsible messages when these are conveyed via entertainment media, where the "selling" of the behaviour is less obvious [66]. Stories offer an effective way to influence attitudes and behaviour because viewers are involved with their narrative structure [44]. The involvement with the narrative is often called transportation [28]. Green and Brock [27] demonstrated greater attitude change among readers who were "transported" into the narrative world. Moreover, Weber [74] promotes storytelling as an essential element for experience design and a method to communicate the city experience 
to a tourist. The use of a story or of a character increases the audience connection and engagement, and it can help in meaning-making and mediation of touristic sites or events [52].

Recent trends position Transmedia Storytelling as a prime tool in the tourism context, particularly suited to promote locations and their cultural heritage [21]. For example, in New Mexico, USA, The Roswell Experience transmedia experience is told across 32 different city locations using a fictional alien character to introduce visitors to the area's history [59]. The experience brought a fresh way to experience the city's rich and unusual heritage by involving the small town business and local inhabitants in it. Which resulted in a marketing campaign that brought visitors from outside to the city to engage with the transmedia experience.

Bear 71 is a multi-platform interactive social narrative created by the National Film Board of Canada [41]. Participants explore and engage with the world of a female grizzly bear through webcams, augmented reality, geolocation tracking, motion sensors, social media, and other platforms and channels. The Bear 71 experience was one of the first TS experiences delving into the intersection of humans, nature and technology raising awareness towards the impact of human intervention in wildlife in a story told through the eyes of the female bear.

Recently in Portugal, the TS TravelPlot Porto publicized local brands while informing and entertaining tourists at the same time [22]. Through the eyes of a character named Peter Smith, visitors learn about highlights of the city. Several platforms and channels were used to communicate the story: a mobile application (iPhone/Android), a website, a print map, live events (gastronomy, wine tasting, a Douro cruise), and social networks (Twitter, Pinterest, YouTube). TravelPlot was one of the TS experiences designed from scratch to be a TS to be experienced by tourists, this work showcases the hardships of bringing together all the different elements at the service of such a demanding audience. Furthermore, it exposes the difficulties in evaluating such experiences and distilling design guidelines based on that.

Moreover, the Transmedia interactive documentaries Welcome to Pine Point [75], Safe Beaches, Shellfish, and You (SBS\&Y) [61] and Miners Walk [43] are TS projects that were not purposefully created with tourists in mind, but they both provide insightful experiences regarding locations (PinePoint city, Newhampshire Main and Ijen Crater) and allows for diverse approaches to sense-making of a place. Both interactive works connect journalism with critical performance to address complex sustainability issues through collaboration between diverse public participants. Welcome to Pine Point explores the aftermath of the mine's closure, including residents' loss of their homes, the national diaspora and the residual environmental damage left. A range of transmedia techniques (poetry, interviews, photos, videos, visual reconstructions) provides a rich space for readers to co-perform the experience of living in Pine Point [75]. The project Safe Beaches, Shellfish, and You (SBS\&Y) combines written reporting with photography, documentary audiovisual material, interactive graphics, and immersive experiences among which "photospheres," images of 5 beaches in New Hampshire and Maine presented through scrollable 360degree imagery enhanced with an audio piece of the sounds at the beach [61]. Finally, the Miner's Walk explores merging short and long form video with an online interactive experience showing how Ijen Crater has become one of the few places in the world where miners, the sulphur industry, and tourism exist side-by-side in uneasy fellowship. As the experience unfolds the audience gets to know the miners themselves, the impact of technology is having in the location, and how tourism is shaping the miners future [43]. Together, these interactive documentaries showcase how we can provide opportunities for the audience to imagine themselves at these locations with the purpose to allow for the co-creation of a lived sense of understanding.

As Pratten highlights, "stories that matter when told to the right people at the right time" [60]. In our project, we leverage on the power of TS to fully immerse and emotionally engage 
visitors with the local destination values. Our artifact is novel in combining the power of fictional storytelling delivered through a location aware multimedia story with a web documentary about the local culture and values.

The TS examples found in literature, have a experimental nature and have been seldom evaluated or documented in details [59] [41]. The TS design features of such projects remain quite obscure making hard for others to learn and replicate such experiences. In addition, some of these works do not report on user evaluations, or were evaluated with using a limited convenience sampling method and not with tourists in the real-world context [22]. Over the next section, we describe in detail our design and evaluation endeavors hoping that these findings will help others in designing and extending the knowledge on TS experiences.

\section{Designing fragments of fragments of Laura Transmedia story}

While thousands of tourists visit the island of Madeira every year, many of them overlook its historical value in the panorama of the European and Portuguese times of the Discoveries, and the existence of what is one of the most unique ecosystems in Europe: the UNESCO World Heritage Laurisilva Forest, a primary or old-growth forest, with many trees predating human settlement (c. 1420) [37]. The project followed a Research Through Design [80] experimental development process. The TS experience was conceived as two pronged. One one side, the FoL location aware multimedia story (LAMS) to leverage on the potential of narrative persuasion and character involvement [28] while on the other hand the FoL Hypermedia Platform (HP) to foster an authentic connection between tourists and locals, stimulating understanding and emotional involvement $[16,67]$ ( see Fig. 1).

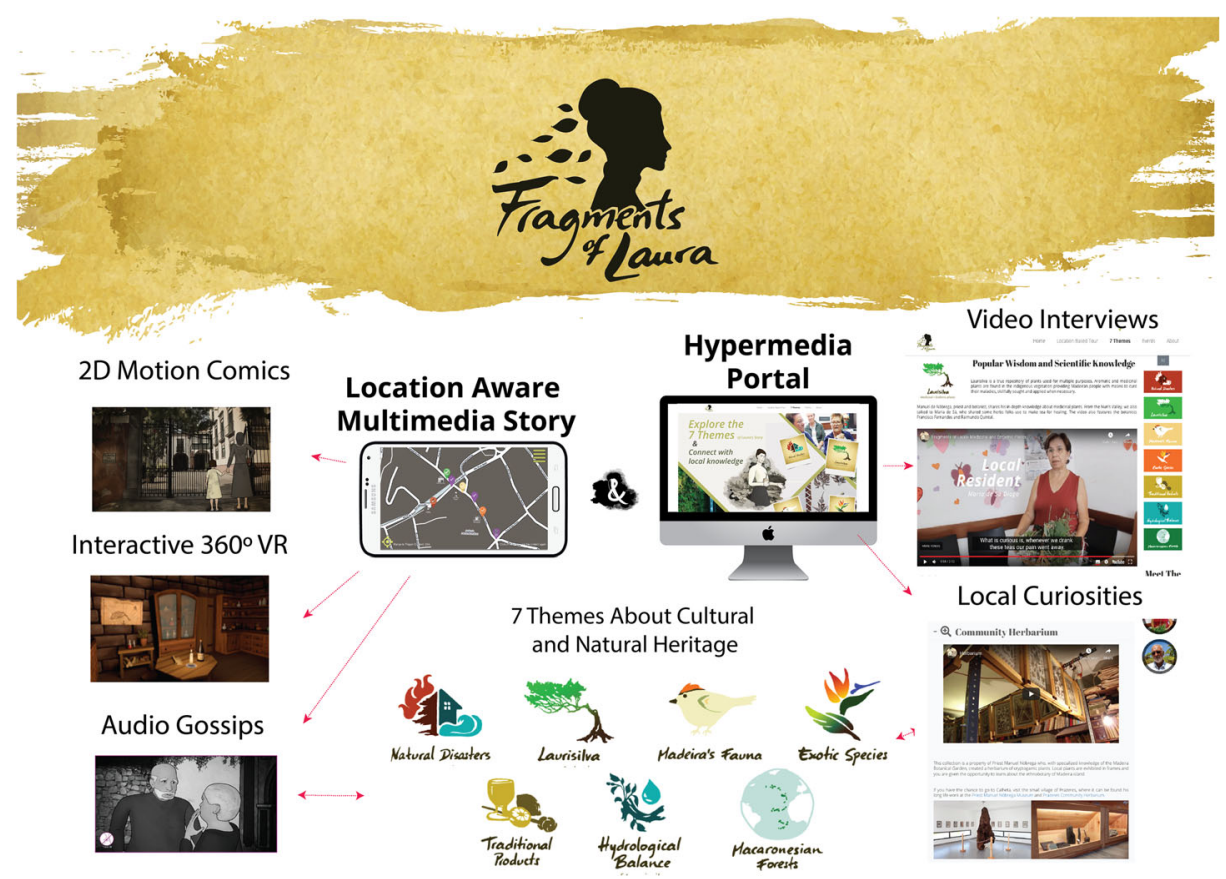

Fig. 1 Illustration of the two main components of Fragments of Laura TS experience: the FoL LAMS and the FoL Hypermedia Platform. The TS content relates to themes regarding Madeira local values and heritage 
The experience was designed to satisfy a broad tourist segment, targeting national as well as international tourists, spanning from young to mature adults who are tech-savvy, seeking for enjoyment and adventure, in a out of the box experience that allows them to get in touch with the local community and values through a different approach.

Previous work [19] paved the way to the TS experience presented and discussed in this article.

\subsection{The narrative plot}

The FoL fictional story follows a classic Aristotelian dramatic arc, where the protagonist undergoes a series of adventures and challenges culminating in a significant event and transformation of the character. The events are set in the nineteenth century, touching on historical events of the island and its ecosystem including the medicinal uses (presented as folk remedies) and other benefits of local flora. Further inspired by Greek tragedy, we looked at the function and model of the Greek Chorus. The Greek Chorus is a group of performers who comment and provide background information on actions and events that are taking place in the story to help the audience follow the performance. By doing this, the Greek Chorus creates a deeper connection between the characters and the audience. Furthermore, the Chorus would allow the playwright to create a kind of literary complexity only achievable by a literary device controlling the atmosphere and expectations of the audience [36].

The FoL narrative invites the audience to embark on a physical quest to follow its heroine, Laura Silva. Orphaned by the historical floods that crippled the island in 1803 Laura escapes a difficult childhood in a nun's ruled orphanage, to study abroad as a naturalist. With a unique talent and passion for taking care of nature, eventually, she returns to Madeira to pursue her life's work studying, cataloguing, and defending the local Laurisilva, Madeira's forest. Her notes and samples are kept in a vast herbarium - an annotated and illustrated book of medicinal plants, nonetheless, her work, ahead for the times, is not well received by everybody in the small island community and often mistaken for witcheraft.

By following this narrative, the audience learns about the history of the island, enters several iconic buildings and monasteries, and discovers how various local plants can be used as remedies while the natural capital of the forest was being degraded and exploited. The fictional story is based on the historical events that occurred during the times of this massive exploitation, weaving science, traditions and folklore of the island. Despite being set in the nineteenth century, many of the situations the main character faces, such as natural disasters, invasive species and the endangered natural patrimony of the island are still relevant to our times (see Fig. 2). By linking the proposed fiction with the current reality, our goal is to entice the audience to contemplate the richness of the island and reflect on the sustainability of its patrimony.

\subsection{The Transmedia story experience design}

In order to create and unified and coordinated TS experience FoL was accurately designed to span several different media and platforms (see Fig. 1). On the one hand the fictional narrative was adapted to work as a Location-Aware Multimedia Story (FoL LAMS), where the audience is guided through the exploration of the physical streets of Funchal city. As the audience unravels the drama while gaining knowledge about the history and the natural heritage of the island. Touchpoints between the story and the audience were designed with attention to the 


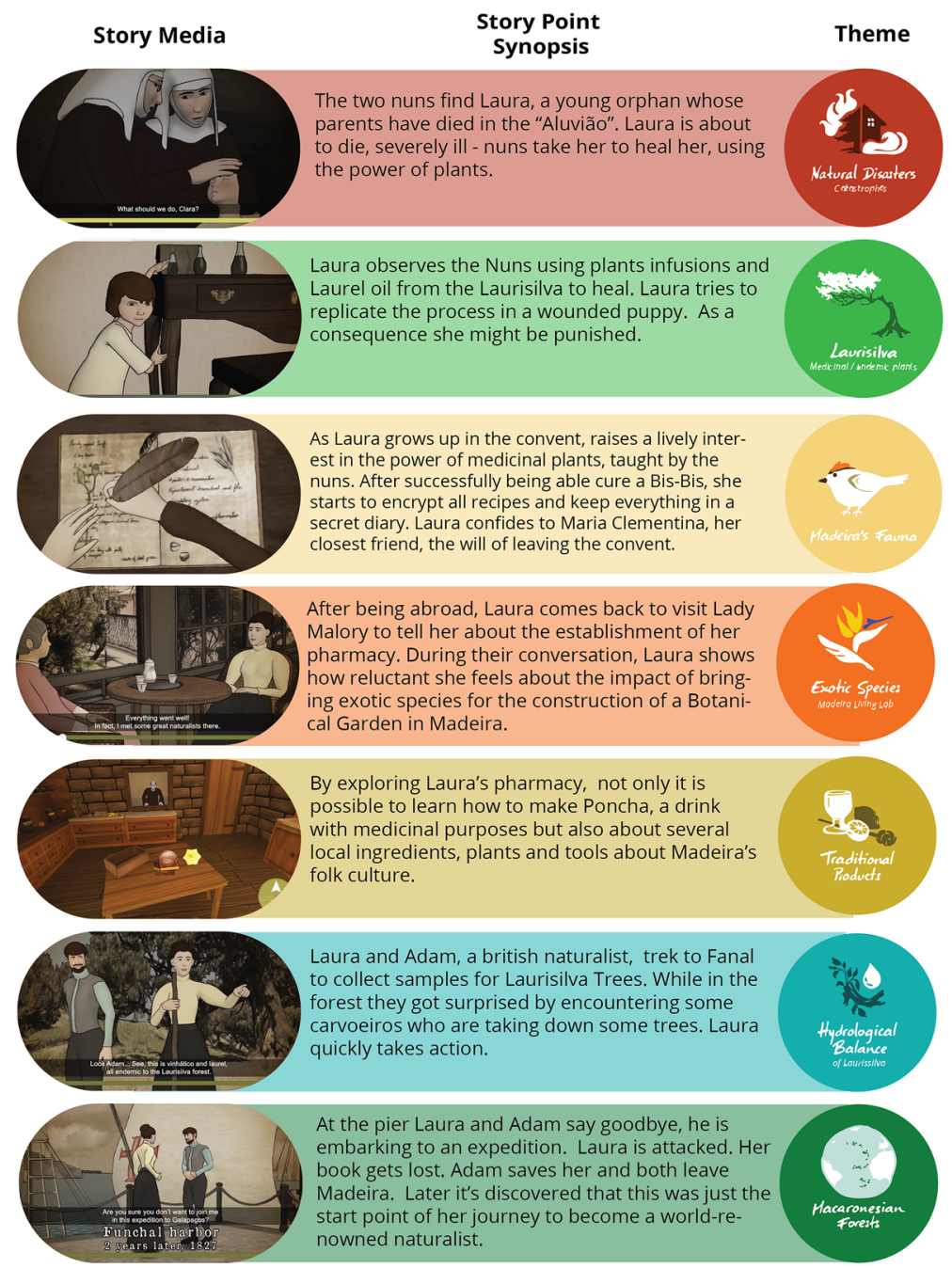

Fig. 2 Summary of the seven episodes of FoL fictional. On the left, a screenshot of a frame of the animated episode, in the middle the episode short synopsis, and on the right the theme which the episode touches on

weaving of historical information, information about the island's natural capital, the progression of the plot and exploration of the physical and architectural settings. Guided by the design of conventional Location-Aware Multimedia Story [50], FoL LAMS is delivered through a map driven mobile application, where Laura's story is highlighted in sequential order by icons indicating meaningful locations that the audience needs to visit in order to collect story content. Each location is associated with a story point, and its icon is representative of this association (see Fig. 3 - Top Right Corner). Participants must find the desired locations by walking to it, guided by the mobile phone Geo Positioning System (GPS) (see Fig. 3 - Top Left Conner). The mobile device vibrates upon reaching the correct location, and the story content is made available. On the other hand, the FoL HP was designed to complement the fiction providing visitors with the contemporary local community knowledge and opinions about their history, heritage and values. The FoL HP was conceived as a further channel of the 


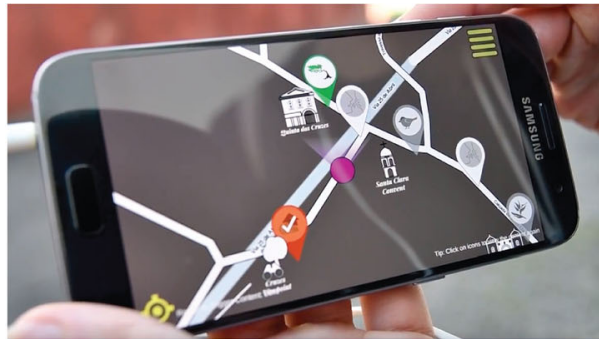

How to discover the story of Laura Silva:

3. The story fragments are sequential and GPS activated. They unlock when you are close to them!

4. Once you watched the story fragment go to the next location

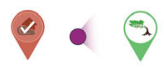

How to discover the story of Laura Silva:

1. It is spread around iconic landmarks of Funchal

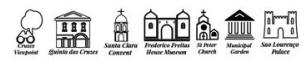

2. Follow the map to uncover all the story fragments. There are 7 !

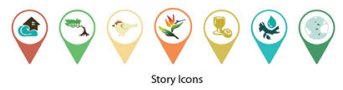

How to discover the story of Laura Silva:

5. In between the story, there are Audio Gossips.

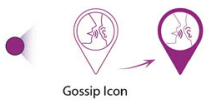

Pay attention to them, they give extra juicy details.

Fig. 3 Top Left: FoL LAMS map-based interface. The participant position is shown as a purple dot, while the different story points and landmarks are shown as colored and grey icons. Top Right and Bottom right and left: Screenshots of the mobile application tutorial, explaining the interface details and how the experience develops

TS, to connect the dramatic events of the fiction, with current facts and value at play in the local community. In the next sections, we describe in detail all of the components and media touchpoints of the FoL TS experience.

\subsection{The fragments of Laura location-aware multimedia story (FoL LAMS) touch points}

Several differently mediated touchpoints compose the FoL LAMS: six two-dimensional (2D) Motion Comics; one Interactive $360^{\circ}$ Virtual Reality (VR) reconstruction of Laura's pharmacy, six Audio Gossips (distributed along the main story path), and 12 Multimedia Pop-up Windows, appearing at the beginning and the end of each touchpoint. The rest of this section describes in detail each touchpoint and its features.

2D motion comics The fictional story of Laura is narrated in six Motion Comic episodes (the combination of audio-visual elements and 2D animations). Through the episodes a series of historical and scientific facts about the island are exposed: such as the floods of the 1803, the destruction of the forest by the local timbermen, the visit of notorious scientists such as Charles Darwin to the island). The main character transforms along the story, from an orphan victim of the flood, to an affirmed and courageous scientist. The story plot and character emotional journey are designed to win over the audience, make them care about Laura and her cause, transporting them closer to local values and history (Fig. 4).

Interactive $360^{\circ}$ VR Laura medicinal pharmacy is rendered in $360^{\circ} \mathrm{VR}$ and can be navigated similarly to a 3D computer game (see Fig. 5). The audience embodies the character of Laura and must explore the virtual environment to find the right ingredients to make a traditional local remedy (see Fig. 5 - Left). During this process, the participant is informed about the qualities and benefits of several endemic products and artefacts. Through the Interactive $360^{\circ}$ 

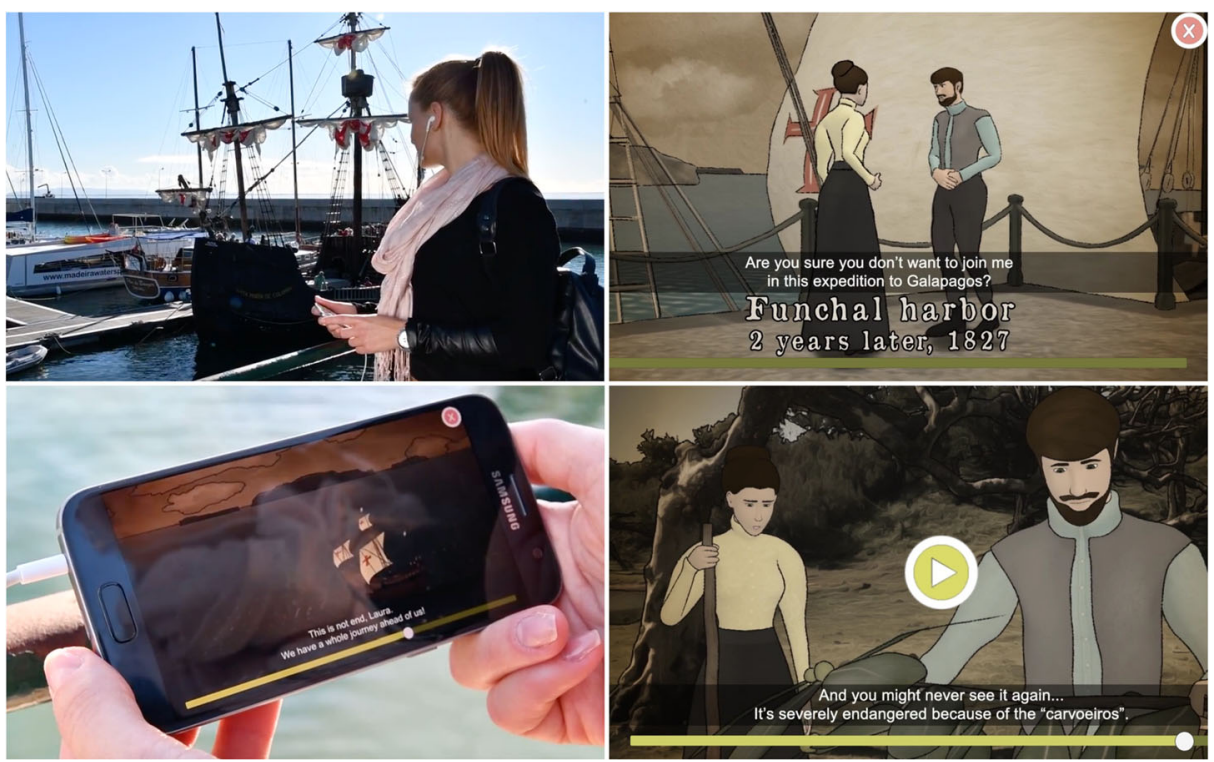

Fig. 4 Upper Right: Participant in Funchal Harbour, the last location of FoL LAMS. A replica from a Portuguese Caravel is visible in the background; Upper Left: Screenshot from the last episode of the mobile fiction when Laura embarks on a Caravel, from Funchal Harbour; Lower Left: Mobile phone screen visualizing the 2D Motion Comic; Lower Right: Screenshot from the of the 2D Motion Comic, waiting to be played

VR touchpoint, the participants are stimulated to take action and assume Laura's role as a pharmacist for a few minutes. Moreover, they can experience the accurate reconstruction of an original Madeiran pharmacy environment in the nineteenth century.
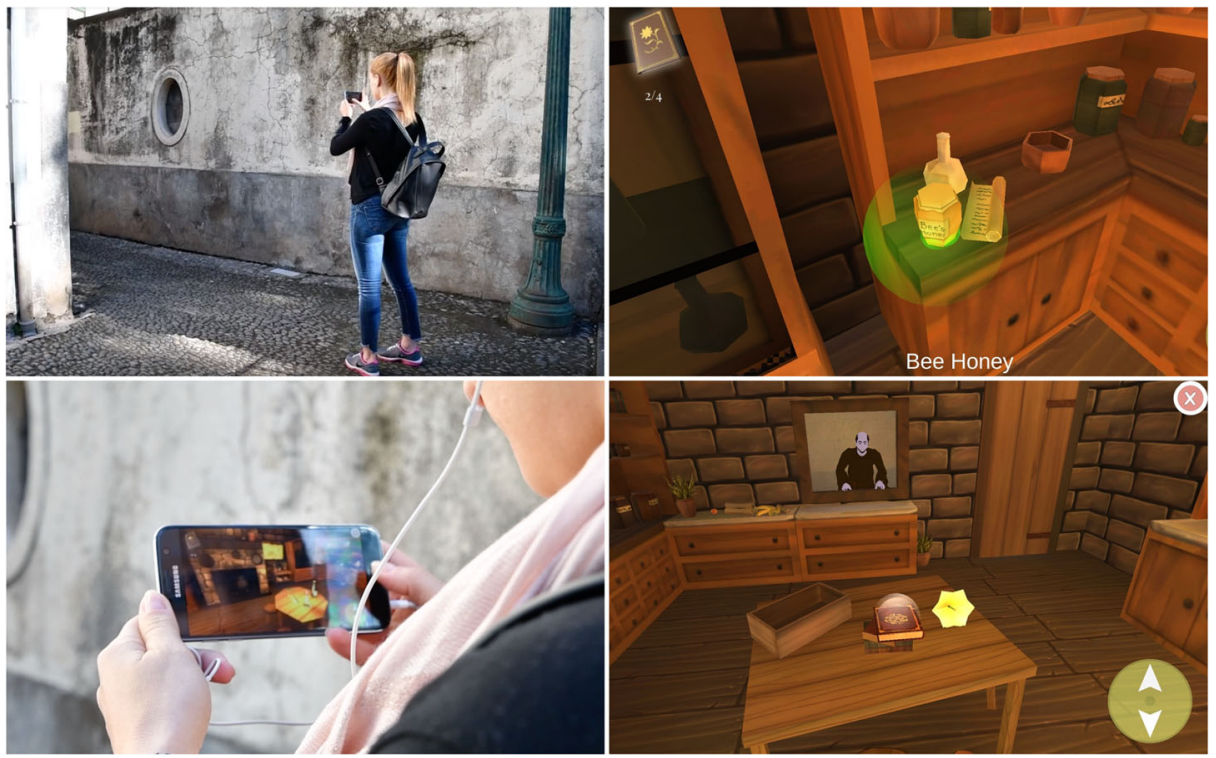

Fig. 5 Showcase of the Interactive $360^{\circ}$ VR touchpoint: "Laura's Pharmacy". Left: Participant on the touchpoint location holding the mobile phone. Right: Screenshots showcasing "The Pharmacy" VR environment where is possible to see some of the interactable objects 
Audio gossips Six audio clips, inspired by the narrative device of the Greek Chorus, were designed and distributed across the story path to fill in background details of the story. This media is meant to recreate the experience of eavesdropping on the community gossiping. Its narrative goal is to fill in background details for the action, the characters back story and foreshadow events. The Audio Gossips features audio over a static black and white image of two locals talking to each other. The fact that the image is static, facilitates the participants to look around and take in the architectural layout and monuments, rather than the screen. The Audio Gossips are not essential to comprehend the story; instead they complete it and give perspective, and can be skipped simply by pressing a button (see Fig. 6).

Multimedia pop-up windows This feature integrates the fiction with facts and curiosities about the place. There are two different kinds of multimedia pop-ups: short texts, displayed before the story clip, to highlight interesting elements of the location surrounding the audience; and audio-visual after the story clip had finished playing, which presents short previews of the video interviews with locals, synthesized from the FoL HP (see Fig. 7). The participant can choose to watch or save it for later viewing (see Fig. 7Right). Furthermore, the full version of the interviews can be followed in the FoL HP.

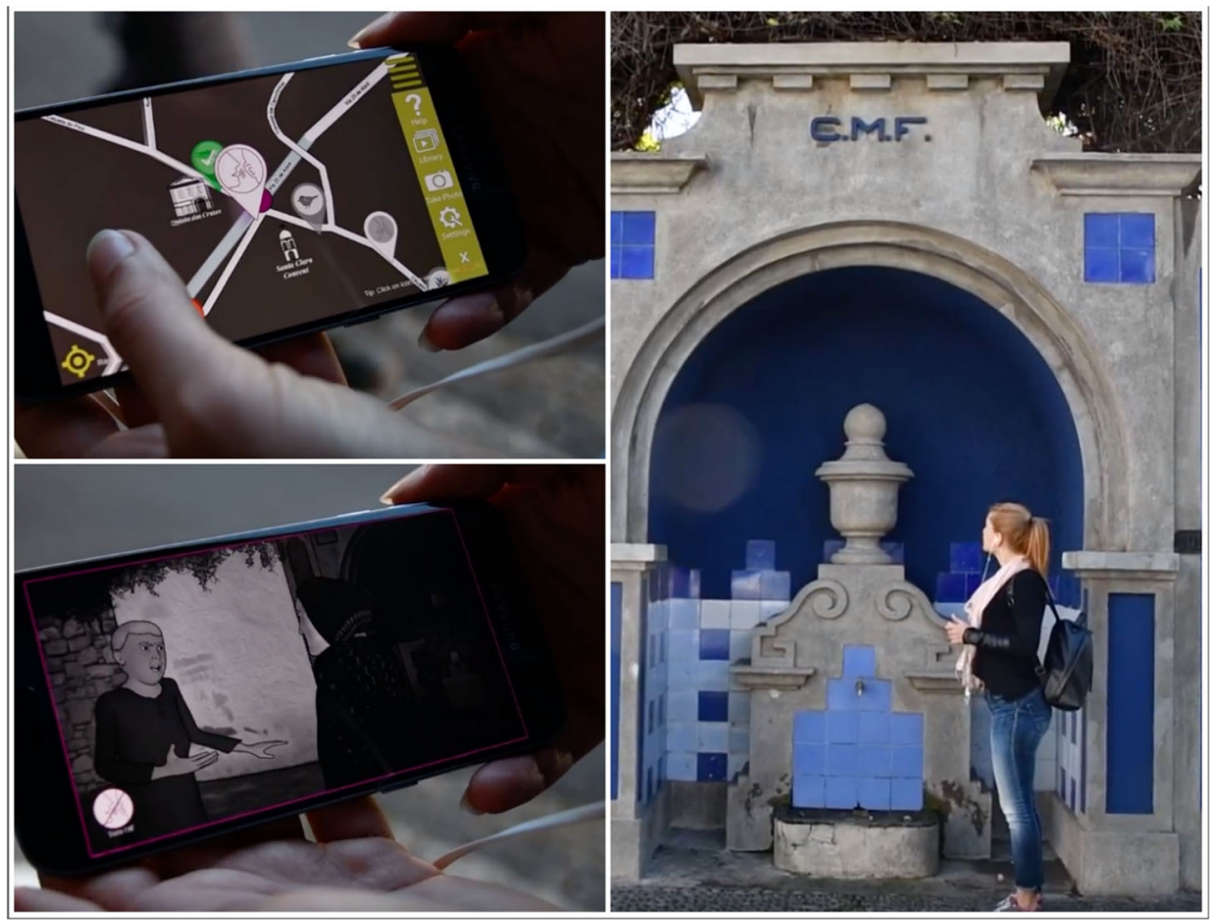

Fig. 6 Participant interacting with one Audio Gossip touchpoint. Left Top: Screenshot showcasing the audio gossip icon highlighted; Left Bottom: Screenshot of the audio gossip image. Right: Participant observing the fountain where the gossip was exchanged, while listening to it 


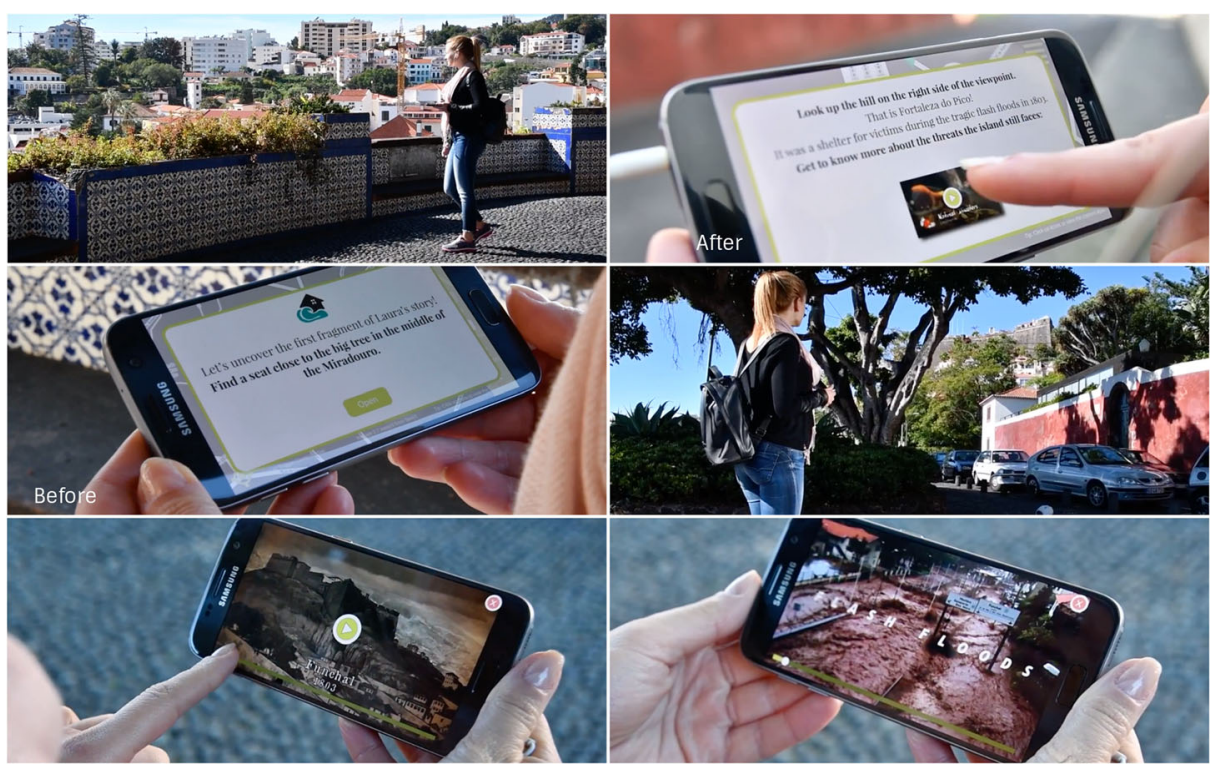

Fig. 7 Examples of the Multimedia Pop-up Windows; Left: Multimedia Pop-up Window before the episode, indicating to the participants where they can sit and watch the story comfortably. Right: Multimedia Pop-up after the episode, bringing awareness to the Fortress, up in the hill, that is featured in the story clip

\subsection{The fragments of Laura hypermedia platform (FoL HP) as a repository of local knowledge and opportunity for tourist to know locals}

The FoL HP is designed to enrich the viewer's knowledge about local heritage while providing opportunities to get to know the island's inhabitants better. The platform functions as a repository of locally collected video interviews highlighting many aspects of Madeiran island's natural capital and local culture. A team of three researchers interviewed 18 local sources collecting around $20 \mathrm{~h}$ of video. The interviewees were chosen according to: their expertise and local knowledge on the themes, availability to be interviewed and willingness to contribute to the project. The participants were not remunerated for their contributions. The platform was initially designed as a standalone, independent channel, with its own identity [20, 51, 65], while thematically and technologically connected to the fictional TS component. In this way, tourists were not required to experience the LAMS in order to enjoy the community interviews web platform, and vice versa. However, after several pilot evaluations and participatory sessions with local community members and visitors to the island, pointed out at some lack of cohesion among the two TS components as a result, the separation was dropped. Therefore, the final redesign of the hypermedia platform, resulted in a unified experience between the fictional story and local interviews, under the same visual language and name. In this article we refer to the last unified version of the FoL TS components.

In the FoL HP homepage, is designed to introduce to tourists the concept of TS experience by showcasing a promotional video of the FoL LAMS and FoL HP (see Fig. 8-2,3). The top of the page contains a navigation menu, with five items: Home, Location-based Tour, 7 Themes, Events, About. The Location-based Tour and the 7 Themes each have a drop-down menu (see 


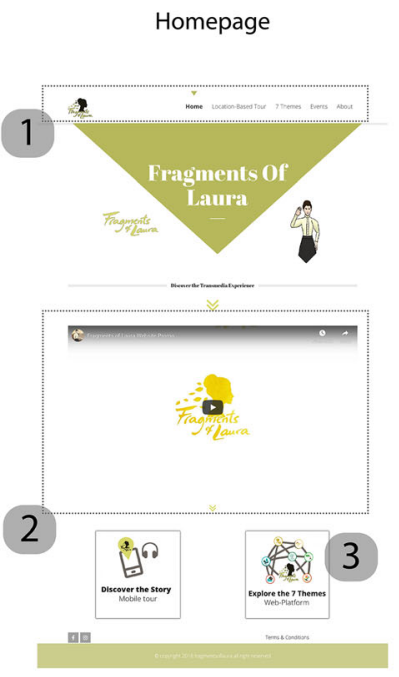

\section{Explore 7 Themes Web-Page}

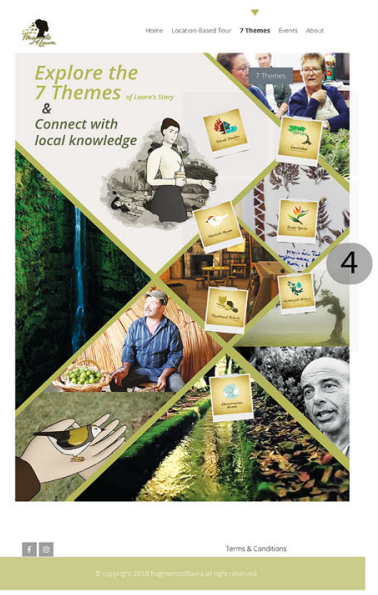

Laurisilva Theme Web-Page

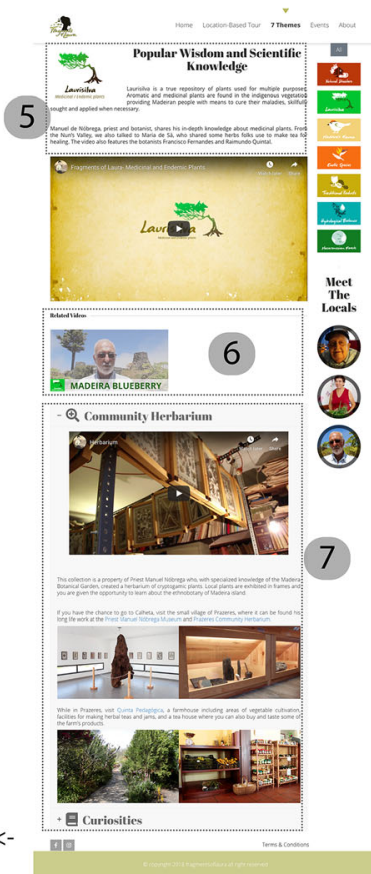

Fig. 8 Screenshots illustrating three web pages from FoL Hypermedia Platform; Left: the FoL HP Home page; Middle: 7 Themes web-page; Right: The Laurisilva (Medicinal and Endemic Plants) web page

Fig. 8-1). In the 7 Themes web page participants can choose to browse the themes by clicking one of the seven polaroids shaped icons (see Fig. 8-4). The theme page outline is identical for all 7 themes. As an example, the Laurisilva theme web page features a text summary of the video clip at the top, followed by the video clip of the interview, and finally a list of related videos (see Fig. 8-5,6,7). Further information in mixed-media format (images, text, maps, photo galleries, schemes and infographics) is proposed at the end of the page. The content and media of this final section, is tailored to the selected theme. Its goal is to either propose onsite activities, locations to visit or local products to engage with. Through the FoL HP locals share assertive information on respectful behaviours towards the local destination. To bring the fictional story close to the real-world facts, some fictional characters feature on the FoL HP webpages.

The FoL HP is further linked with the mobile fiction, over seven specific themes (see Fig. 9). We describe the seven themes in detail below.

Natural disasters This theme describes how wildfires and floods can harm Madeira's environment. The local scientists explain the nature of flash floods and wildfires, and how to prevent them. This theme links back to the narrative first episode, when Laura is introduced, orphaned by one of the most significant floods in the island in 1801 .

Laurisilva (medicinal and endemic plants) Aromatic and medicinal plants are abundant in the indigenous vegetation of the island. Under this theme, locals share their in-depth knowledge about medicinal plants and how they can be used to make healing infusions. This thematic is reflected in 


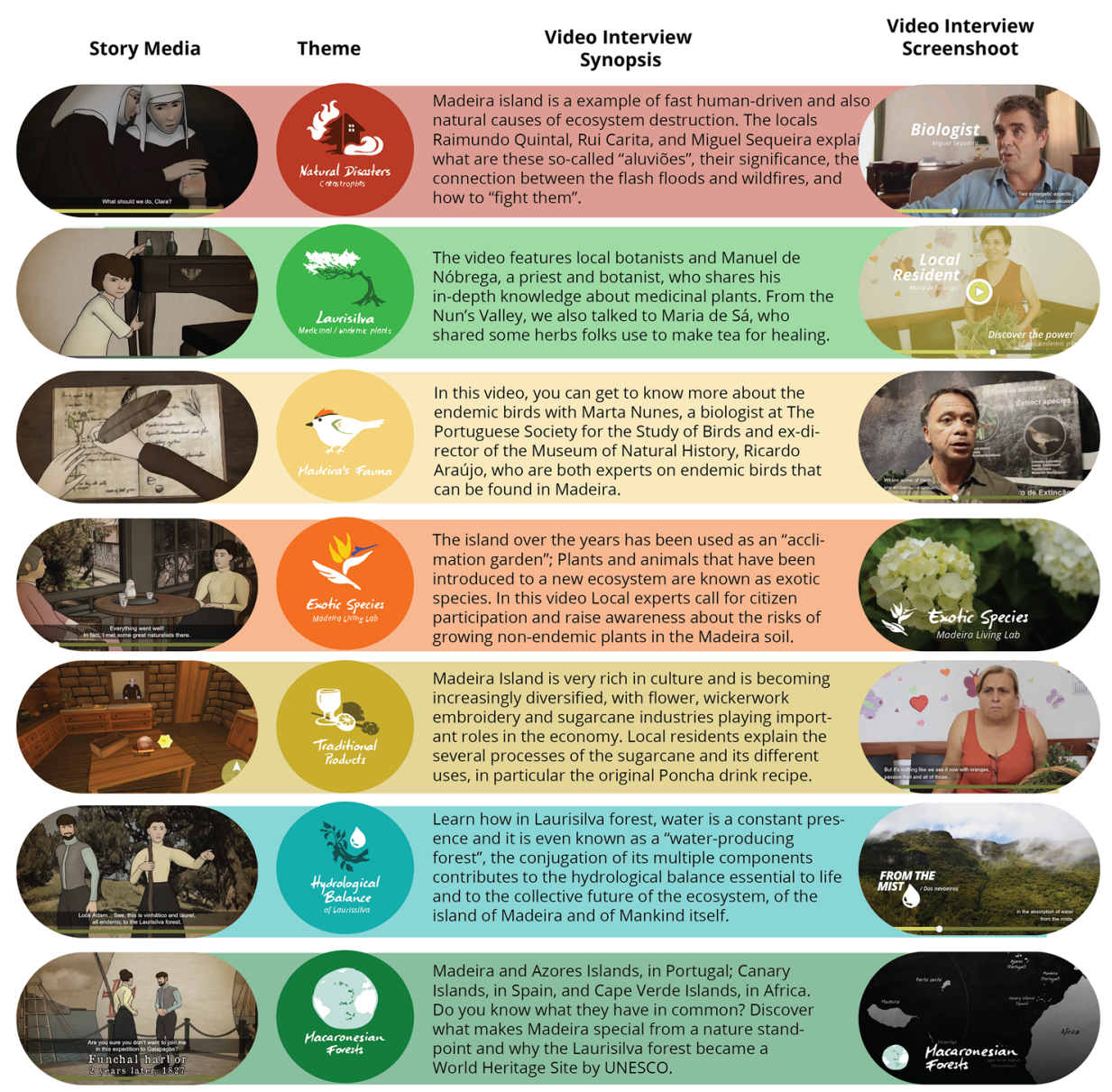

Fig. 9 Connection between the web platform video interviews and the fictional narrative

the second episode of the narrative when Laura witnesses a nun treating a neighbour with medicinal plants, and eventually puts this knowledge in action herself.

Exotic species This theme presents the problem of invasive exotic species that can threaten the local biodiversity. In the fictional narrative, Laura, after studying abroad, is bothered and reluctant regarding the project for a local botanical garden which would bring invasive exotic species to the island and endanger its indigenous species.

Traditional products One of the most important activities in the nineteenth century was the transformation of sugar cane into sugar and alcoholic beverages. Residents explain the different uses of the sugar cane, including the original "Poncha" drink recipe. In the fiction, specifically in the $360^{\circ} \mathrm{VR}$ pharmacy, the audience learns about several local ingredients and folk traditions, including the "Poncha" local drink and remedy. 
Hydrological balance This theme is concerned with the importance of the local forest for the island's delicate hydrological balance. In one of the fictional story episodes Laura and her naturalist friend are walking in the forest, talking about how the trees capture water from the mists.

Macaronesian forests This theme highlights how the Macaronesian islands, of which Madeira is part of, are considered as one of the most important biodiversity centres worldwide. In the last episode of the narrative, Laura is forced to embark on a journey bound to a different Macaronesian island, which holds similar characteristics to Madeira.

\subsection{Technical implementation of fragments of Laura Transmedia storytelling}

Regarding technical implementation of FoL LAMS, the mobile application was programmed in $\mathrm{C \#}$, using the Unity game engine. The main interface is composed of a map which uses a Mapbox Api ${ }^{2}$ customizable design, overlaid with clickable buttons for each story point. Since the story is location dependent, the map view acts as the main navigational aid of the transmedia experience. GPS positioning (which identifies the location of the user) and a mobile data connection (for map tile download), point the user to the right path to collect the story content. In the interactive $360^{\circ} \mathrm{VR}$ scene, the $3 \mathrm{D}$ environment was built using $3 \mathrm{DS}$ Max Studio and later incorporated in Unity as well. The rotation and orientation in the virtual environment is provided by the Google Cardboard Unity plugin ${ }^{3}$ upon receiving the input from the mobile accelerometer and gyroscope. The sequence of story events within the pharmacy is handled with the help of the open source tool Fungus. ${ }^{4}$ All multimedia content is stored in the device and no mobile data connection is required after the map is loaded.

Finally, the FoL HP interface was deployed in a customized WordPress template.

\section{Evaluation of fragments of Laura Transmedia experience}

FoL TS was designed to investigate how TS can be used to emotionally engage visitors with the local destination, bring awareness, and connections between them and the local community. Three research questions (RQ) guided the FoL TS evaluation:

- RQ1: Can FoL TS provide a fulfilling and memorable tourism experience?

- RQ2: Did both FoL TS components supported tourists to emotionally connect with the island local values and community?

- RQ3: Did FoL TS enriched the knowledge about the local destination cultural and natural heritage in a significant way? If so, what role did the two different TS components play out?

To answer RQ1, a set of questions were applied at the end of the FoL TS experience related explicitly with the tourism experience. To address RQ2, the FoL LAMS and FoL Hypermedia

\footnotetext{
$\overline{2}$ https://docs.mapbox.com/unity/maps/overview/

3 https://arvr.google.com/cardboard/developers/

4 https://fungusgames.com/games/
} 
had to be evaluated separately. Hence, the evaluation included questionnaires tailored and applied right after each of the components.

Finally, to ensure that RQ3 was addressed, the evaluation included questions regarding the participants' perception of acquired knowledge, and a quantification of the amount of acquired knowledge before the FoL TS and after. Details on the evaluation procedure, measures, and how they related to the research questions are included below, followed by the description of the participants' sample, quantitative and qualitative results.

\subsection{Evaluation procedure}

Participants were made aware of FoL TS and recruited for the study through events on social media, posters and flyers distributed in hotels, Airbnb lettings, hostels, and some tourist points around the city of Funchal. The goal was to evaluate the FoL TS experience in the closest way to a real tourism experience. Therefore, participants had the freedom to choose what would be the most convenient way for them to experience FoL. It was mentioned in the sign-up form that the experience would be best done either individually ( 1 participant, one mobile phone) or in pairs ( 2 participants sharing a phone) but bigger groups were welcomed with the constraint of a maximum of 6 participants per session, due to available devices and experimenters.

The whole protocol, comprising 6 phases, lasted for around $1 \mathrm{~h} 30 \mathrm{~m}$. The number of experimenters per session depended on the group size, with one experimenter per pair of participants. Figure 10 presents and summarizes the protocol phases divided into steps.

Experimenters met the participants at the physical location where the FoL LAMS starts to explain the research procedure. Experimenters asked the participants to sign the consent form and administered the first questionnaire (Q1_Demographics). Q1 consisted of questions regarding demographics and the Madeira Questionnaire Scale (MQS) created to test their general knowledge about Madeira. Experimenters provided participants with Samsung S7 and S7 Edge mobile phones and headphones. On average, the duration of the FoL LAMS lasted around $45 \mathrm{~min}$, after which participants were asked to complete the second questionnaire

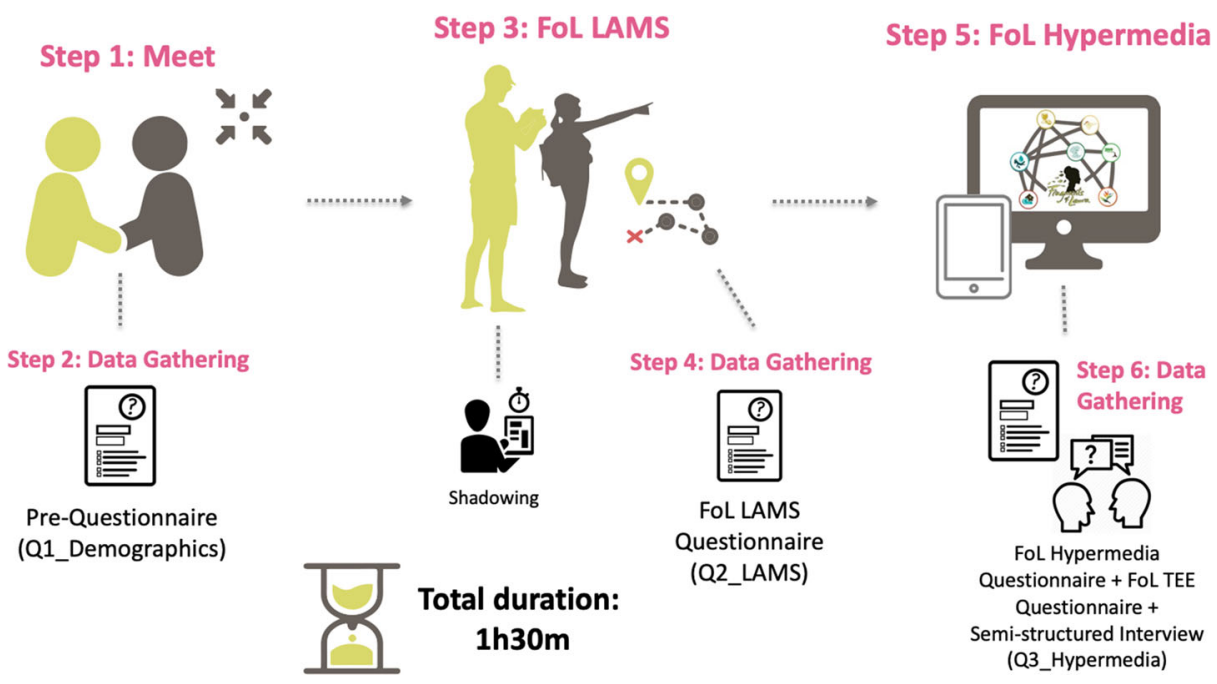

Fig. 10 Overview of the steps composing the FoL TS evaluation protocol 
(Q2_LAMS). Q2 included general questions about the experience with FoL LAMS and the post experience MQS. Afterwards, participants were asked to browse the FoL Hypermedia for at least $10 \mathrm{~min}$. A specific questionnaire was administered after this last step (Q3_Hypermedia). Q3 asked questions about the specific experience with the FoL Hypermedia, about the overall FoL TS and again the MQS.

Finally, a 5 to $10 \mathrm{~min}$ semi-structured interview was conducted and audio-recorded. Participants were rewarded with a unique cotton bag printed with the FoL logo and some leaves from the local flora (see Fig. 11). Due to lack of time, some participants preferred to visualize the FoL HP component later on. These participants received a follow-up e-mail with a link to FoL HP and questionnaire Q3_Hypermedia.

\subsection{Measures and data analysis}

Participants' demographics and a ground truth regarding their knowledge about Madeira culture, before engaging with the FoL TS. Subsequently, we collected data about their experience after each of the TS components (FoL LAMS and FoL HP) separately. To measure narrative transportation, it was essential to understand how involved in the fictional narrative

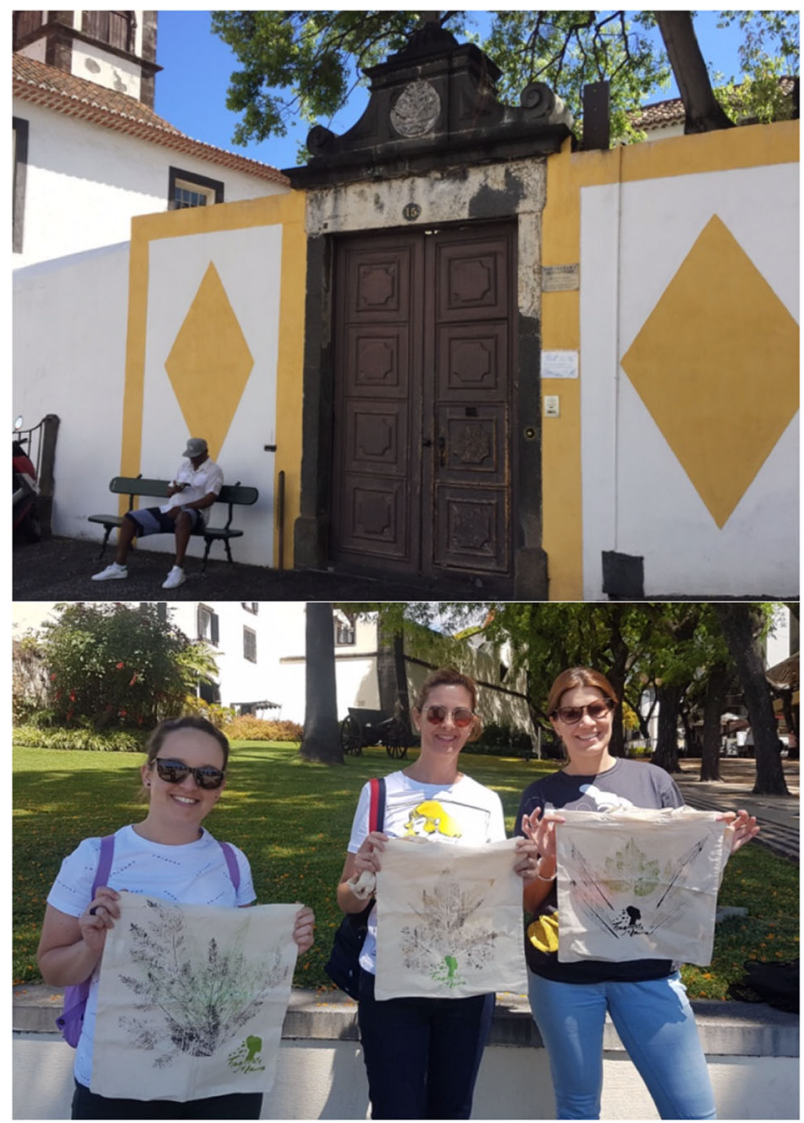

Fig. 11 Top: Tourist in the third touchpoint of the FoL LAMS; Bottom: Group of tourists who participated in the FoL TS evaluation holding their souvenir the FoL TS cotton bag 
visitors were, and what they retained after engaging only with the fictional narrative. Subsequently, the FoL HP was assessed through a separate set of tailored questions. Finally, participants were asked to reflect on the unified FoL TS experience, to understand how the two components worked together. In this way, it is possible to identify how each one of the components contributed to the experience. Finally, the semi-structured interviews probed the users in regard to the overall experience and story memorability. Further details about the questionnaires and the data analysis performed were added as Supplementary Material 1.

\subsection{Participants sample demographics and characterization}

A total of 45 tourists responded to the invitation to participate in the FoL evaluation. From these 45 tourists, 10 completed the first stage (interacting with FoL LAMS) and 35 completed the whole experience (FoL LAMS +FoL HP). Forty participants did the tour in pairs (sharing one mobile device but with individual headphones). Participants' age and nationalities are summarised in Table 1.

Regarding participants' experience with smartphones: 7 reported minimal experience, 17 average and 21 high. Concerning their experience with location-based tours: 25 scored low, 19 average and 1 high. Regarding the lengths of their stay and arrival dates, 9 participants had only been in Madeira for less than 2 days, 11 for 2-3 days, 4 for 3-4 days, 4 for a week, 2 for 2-3 weeks, and 15 for more than a month. When asked about the full duration of the stay, 3 participants were staying for 2-3 days, 11 for 3-4 days, 12 for 1 week, and 19 for a longer period of more than 1 month. The majority of our participants, 28, consider themselves as tourists and appreciated interactions with locals.

\subsection{Results}

This section presents the results from the study, divided into two subsections. The first section report the quantitative results from the three questionnaires, starting by reporting the overall FoL TS results then we describe the results from the FoL components (FoL LAMS and FoL HP). Finally, the last section presents the qualitative results from the thematic analysis of the interviews.

\subsubsection{FoL TS experience quantitative results}

The FoL TS experience was considered by participants to be a memorable tourist experience, based on the high median scores for the total score of the Memorable Tourism Experience [10, 34] (MTE) scale (Mdn:5.46 IQR:2.00). Participants reported high values in the median scores across the six dimensions related to the travel experience. From the different dimensions, it is

Table 1 Participants Age Ranges and Nationalities

\begin{tabular}{lll}
\hline Age Range & $\mathrm{N}^{\circ}$ of Participants & Nationalities $/ \mathrm{N}^{\circ}$ of Participants \\
\hline $18-24$ & 15 & Spanish (17), English (4), PT (3), Romanian (5), \\
$25-34$ & 15 & Polish (3), Italian (3), Finnish (2), German (2), Tagalog (1), \\
$35-44$ & 7 & Bulgarian (1), Georgian (1), Russian (1), French (1), \\
$45-54$ & 6 & Greek (1), Thai (1). \\
$65-74$ & 2 & \\
\hline
\end{tabular}




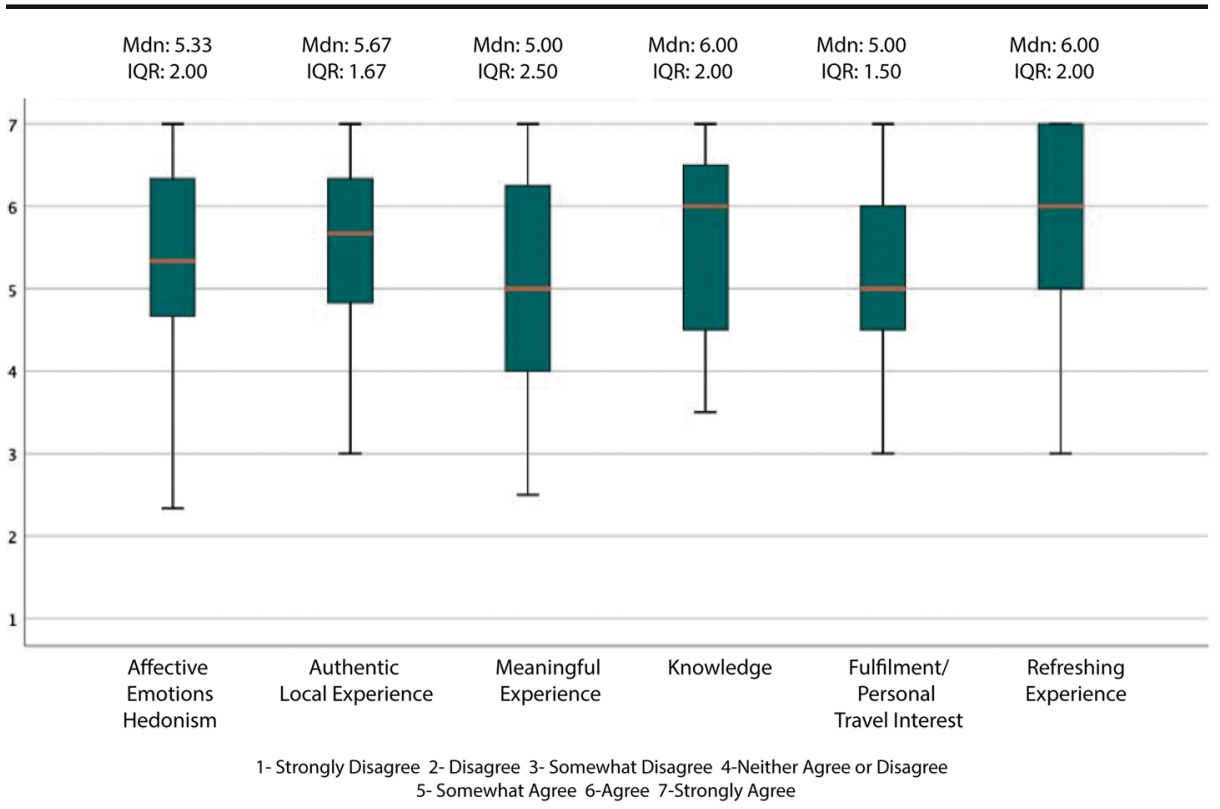

Fig. 12 Results on the six dimensions that compose the Memorable Tourism Experience (MTE) Scale

important to highlight how the FoL TS experience was considered refreshing, authentic, and resulted in knowledge acquisition (see Fig. 12).

In general, visitors had a pleasing user experience with the FoL TS, reporting high median scores for the pragmatic and hedonic dimensions of the User Experience Questionnaire scale [71] (see Supplementary Fig. 1 for median scores and IQR). Participants considered that the level of difficulty and demands were low, however most participants were neutral about recommending the experience to others (See Supplementary Fig. 2).

High median values of the item "The experience enabled me to learn about Madeira Local Values", together with the high median values in the Knowledge item from the MTE scale, testimony for a rich learning experience for visitors. Moreover, visitors' knowledge about Madeiran culture sampled at the three different stages of the experience (before and after FoL LAMS, and again after FoL HP) scored high median values (see Fig. 13).

A Friedman test showed that these differences were statistically significant $\chi 2(2)=44.656$, $p=0.000$. Post hoc analysis with Wilcoxon signed-rank tests was conducted with a Bonferroni correction applied, resulting in a significance level set at $p<0.017$. Furthermore, results were significant with both components of the TS, meaning that each component contributed to increase the participants' knowledge about the island local values. There was a significant difference in the Median scores of the Madeira Question Scale, before participants started the FoL LAMS (MQS1_BeforeFoLAMS) and after they finished the FoL LAMS $(\mathrm{Z}=-3.000$, $p=0.003$ ). A significant difference was also found in the MQS score obtained by the participants before they started the tour (MQS1_BeforeFoLAMS) and in the MQS score by the end of all the FoL TS experience (MQ3_AfterHyper), $(Z=-4.930, p=0.000)$. There was a significant difference when comparing the the participant's median scores, right after the FoL LAMS and after interacting with the FoL HP $(Z=-4.985, \mathrm{p}=0.000)$. 


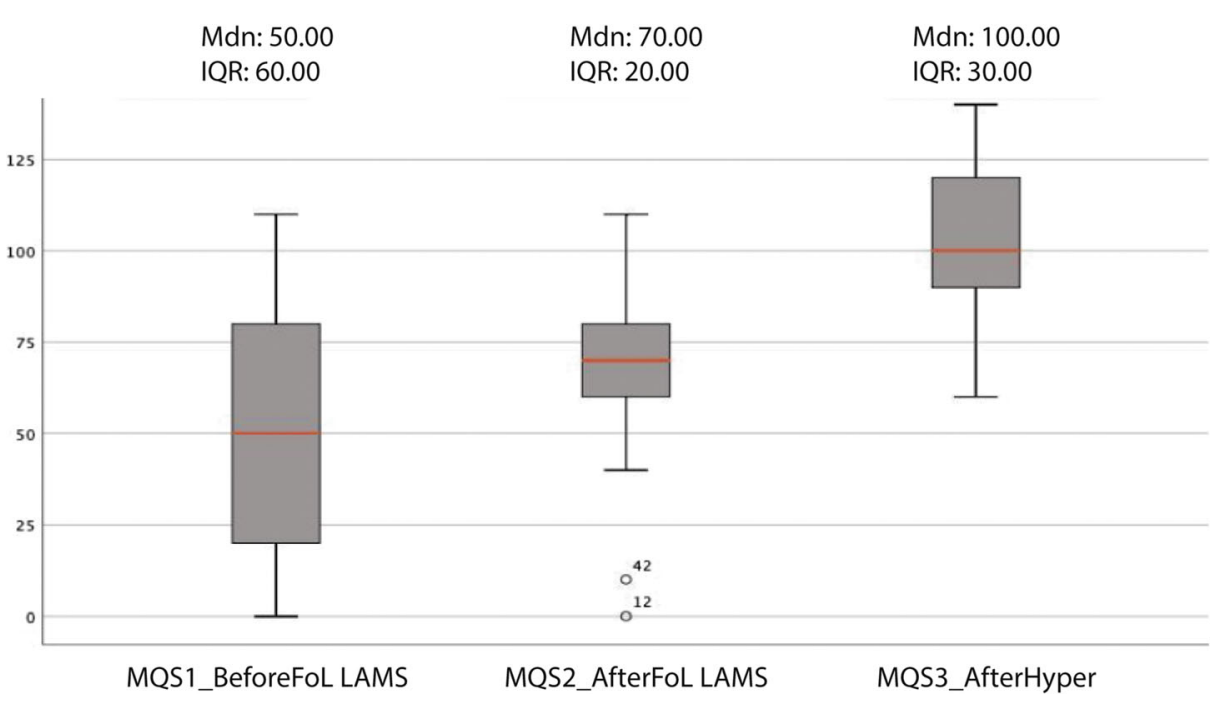

Fig. 13 Median Scores regarding the questions about Madeira local values increased across the three different stages of interaction with FoL TS (MQS1_BeforeFoLAMS: Refers to the median scores before the FoL Tour; MQS2_AfterFoLAMS: After interacting with the FoL LAMS; MQS3_AfterHyper: End of the experience after participants interacted with the FoL HP

\subsubsection{FoL LAMS and FoL HP quantitative results}

The two components of the TS experience were evaluated individually.

Visitors' overall engagement with the FoL LAMS was positive, reporting high median values for the total User Engagement Short Scale [53] (UESS). Looking in detail at the different dimensions of the UESS, most participants reported the FoL LAMS to be rewarding and enjoyed the aesthetics of its graphics (See Supplementary Fig. 3). The FoL LAMS duration was ideal and it was not demanding or difficult to interact with (See Supplementary Fig. 4).

According to the median score for the Narrative Transportation Scale [27], Mdn: 5.00; IQR: 1.44 (on a 1 to 7 scale) most visitors felt quite involved within the fictional narrative, Furthermore, most participants reported high levels of Presence [38] in the narrative, as measured by the item Sense of "Being There" (Mdn: 5.00; IQR: 2.00 (on a 1 to 7 scale) and reinforced by the low median values in real-world awareness (see supplementary Fig. 5).

Regarding the FoL Hypermedia Platform quantitative results, most participants found the FoL HP rewarding, fun and worthwhile the time interacting. It was also perceived as aesthetically and visually appealing and its content served as an enticement to further explore the Island (see supplementary Fig. 6).

Finally, it is important to highlight that the during the data analysis no significant differences were found in the participants perception of FoL according to their demographics (nationality, age range, experience with smartphones and duration of stay). 


\subsubsection{FoL TS experience qualitative results from the participants' interviews}

The 45 interview transcripts were analysed and coded according to a thematic analysis method [7]. The thematic analysis yielded 9 main themes. They are described in detail in the following section. The themes are complemented with illustrative quotes extracted from the participants' interviews. Each quote was assigned a unique identification code (e.g: P0_0). The characters before the " " refer to the participant id to which the quote belongs to and can be consulted in supplementary Table 1.

1) FoL LAMS as an immersive experience: Participants appreciated the immersive qualities of the FoL LAMS. Five participants reported feeling immersed in the experience (P38_1,P39_2), 2 lost track of time (P18_3) and 6 mention that the FoL LAMS duration and walking time was adequate (P36_4). Two participants would have liked the experience to last longer, while one wished it was shorter.

2) Audience appreciation of the fictional narrative: The story (plot, characters and media production) was praised and appreciated by many (P39_12, P10_19, P11_20). Participants enjoyed the story, became immersed and emotionally involved in the narrative (P37_13, P3_14). Nine participants mentioned they were invested in the protagonist's journey, connected with the protagonist at an emotional level, and rooted for her accomplishments. Two participants mentioned how they "worried about Laura" while visualizing the last clips of the story (P9_15); Eight participants, mentioned that her quest to protect nature was important (P30_17). In particular, 1 participant reported being affected by the gossips criticizing Laura (more details in the subsection below: Uniqueness of the Audio Gossips: P34_25). Two participants questioned the open ending of the story and the lack of a "happy ending" (P34_18). Two participants mentioned that they would have preferred a non-linear presentation of the story as they felt it was demanding to follow all the clips in the order established by the authors $\left(P 14 \_20.5, P 13 \_20.6\right)$.

3) The uniqueness of the Audio Gossips: The participants appreciated the Audio Gossips. Five participants specifically mentioned how they enjoyed their unusual and curious content of the gossip, describing it as something unusual and curious (P11_24). Two participants enjoyed how they captured the essence of living in a small location and related that back with their own cultures. Four participants explicitly appreciated how the gossips tied back with some parts of the story (P37_24, P34_25). However, 10 participants lamented the lack of subtitles because the audio was difficult to comprehend, especially in comparison with the other media assets (P37_25, P33_26.5).

4) Multimedia Pop-up Windows as a way to engage the visitors with the physical surroundings and the local community perspective: Two different kinds of multimedia pop-ups enriched the FoL LAMS: before the story, contextualising the audience in the location, and after the story fragment, connecting with the local interviews; both were highly appreciated. Six participants enjoyed how the introductory text pop-ups gave hints regarding where and what to do in the location (P16_27,P17_28). Regarding the poststory pop-ups, 10 participants described them as an excellent complement of the fictional story (P7_29). Participants liked to listen to and connect with the locals as it fueled their curiosity about local issues (P3_30). However, 9 participants reported the synthesis of the video interviews being too short. Participants wanted more factual information $(\boldsymbol{P 1 8} \mathbf{3 1}$, P34_32, P38_33). Two participants mentioned that they skipped the interviews' previews because they wanted to focus on the story and watch the local community content later. 
Two participants explicitly brought up that they might not remember to look up the interviews after the mobile story experience(e.g. look to the website or in the library of the application).

5) The $360^{\circ}$ VR Pharmacy: Participants had very different opinions about the $360^{\circ} \mathrm{VR}$ interactive Pharmacy. For 10 participants it was their favourite touchpoint, the most exciting part of the experience (P36_37,P18_38), while other 7 did not like it (P11_39). Participants who liked it, enjoyed the visuals, exploring and interacting with it. Hesitant participants mentioned that they did not enjoy playing games; therefore, the VR environment modelled after a 3D game, was not appealing for them. Six participants had difficulties in figuring out the interaction strategy (P16_40, P34_42). Four participants proposed that the VR scene being more appealing for a young audience (P20_43). Most participants agreed that the interaction with the $360^{\circ} \mathrm{VR}$ environment could be improved in terms of the instructions given and some interaction aspects.

6) The FoL Hypermedia Platform: All participants appreciated the FoL HP component and how the interviews with the locals allowed them to access facts and scientific content (P14_48). Five participants mention how the fictional story and the interviews with locals strengthen each other (P32_49). Eight participants mentioned how they liked the fictional story but also felt the need to access facts (P17_50,P10_51) while 2 mentioned preferring it over the mobile fiction (P13_47). Fourteen participants appreciated having access to the local community's point of view, and their different perspectives. Participants felt empathy towards the locals and their stories. In general, participants mentioned that the interface, layout and content organization of the FoL HP was easy to follow (P7_52, P3_53). Users enjoyed how the scientific information was delivered in a summarized and engaging form (P15_54). Two participants mentioned that they would have liked more time to fully explore the content of the FoL HP.

7) FoL TS: a learning experience about the local culture: Participants found the FoL TS experience a different and useful way to get to know their destination values. Visitors mentioned learning things that are not normally found in the tourist guides (P34_22; P10_21). Furthermore, 20 participants valued the entertaining way information was presented (through the fictional story or the interviews with locals), different from a long textual description (P31_21.1, P31_21.2). Four participants also mentioned that this was a good way to deliver a first overview of Madeiran culture (P16_23). Participants enjoyed the combination of fictional with the factual and hearing the perspectives of the locals.

8) Suggestions regarding the FoL TS target audience: Some participants expressed concerns about how seniors might not appreciate this technology. At least 4 participants expressed concerns about how elderlies might not be able to interact with it (P16_9). On the other side, 4 participants suggested that this sort of experience would be appealing for a young audience and schools, proposing the addition of gamification elements (P20_10). On a similar note, two participants expressed how the $360^{\circ} \mathrm{VR}$ environment would be cherished by gamers $\left(\boldsymbol{P} 23 \boldsymbol{V} \boldsymbol{F} \_11\right)$. To note that most participants (30) had ages below 44, with the oldest (2) being between 65 and 74 years-old.

9) Opportunities for refinements: Several opportunities for improvements of the TS were highlighted through the interviews. Four participants mentioned that they would appreciate more specific and factual information about the landmarks and locations visited along the tour, how and if the locations mentioned in the story can be visited, what are the costs of tickets, schedule, history (P34_7). Two participants mentioned they would like an even stronger link between the content, application and physical elements in the locations. It was suggested to add more Augmented Reality interactions (P39_8). Interesting to note is 
that at least 6 participants felt rushed to finish the tour because of the research study nature and set up. Some of them wished to spend more time at the locations exploring and even visiting the museums along the way (P9_5, P29_6).

\section{Discussion}

From the evaluation results, FoL TS emerges as a quite successful tool not only in fostering awareness towards the destination and local community heritage but also in providing a memorable touristic experience. Reflecting on the evaluation results and guided by our three main research questions, below, we highlight several takeaways from this study.

When designing TS experiences for tourists, there is much to gain in designing in alignment with the visitors existing needs and desires. However, this is quite a complex task, because tourists are a quite diverse audience; their desires and what they seek for may vary considerably from one tourist segment to another, their age, nationality, and travel motivations. In general, there is a consensus that tourists' desires center around: things and experiences that cannot be found at their home destinations [55], authenticity in their experiences [72]; enjoyment [34], and finally the urge to acquire new knowledge [35]. FoL TS was designed to answer these needs and desires for a broad tourist segment (National as well as international tourists, spanning from young adults to mature adults who are in search of authenticity and urge to acquire new knowledge). One hand the FoL LAMS experience through the exploration of real locations is designed to appeal to tourists seeking enjoyment and adventure, while the Fol HP brings an authentic flavour to the experience, by connecting with its community's opinions. The two components together aim to respond to the tourists desire for new knowledge and understanding of the destination, as well as providing a unique experience tailored to the touristic destination values and heritage.

In response to RQ1 (Can FoL TS provide a fulfilling and memorable tourism experience?), FoL TS was perceived as fulfilling and refreshing, innovative and quite unusual. Looking at the qualitative results of the Memorable Tourism Experience Scale, the FoL TS emerges as satisfying across all dimensions. The FoL TS experience, met the tourist motivations in terms of being an enjoyable and fun experience. Visitors enjoyed the entertaining way information was presented, both in the fictional story and the video interviews. Users highlighted that these formats were more enjoyable than reading long textual information.

Regarding the tourists' desire to acquire new knowledge and understanding of the destinations, the TS was successful in granting tourists' access to authentic and less mundane content that they would not find in traditional communications and information packages produced for tourists.

Hence, we can reply positively to RQ1 by saying that FoL TS succeeded not only as an authentic local experience but generally speaking as a fulfilling and memorable touristic experience. The adoption of new media formats (video, 2D animations and 3D VR), the prompting exploration of the physical locations with the used of the multimedia pop-ups, augmented with fictional and factual information proved to be an engaging strategy to satisfy tourists' curiosity, and amuse them at the same time.

Regarding RQ2 (Did the two components of the FoL TS (FoL LAMS and FoL HP) equally supported tourists to emotionally connect with the island local values and community?), one of our design concerns, was if the two components of the TS could work together to achieve the FoL TS goal. The evaluation confirmed that the two components worked as complementary, 
and respectively brought: satisfaction, engagement and involvement with the local heritage, from the FoL LAMS, as well as empathy,respect towards the local community values and their history the FoL HP.

The FoL LAMS, was for many, the most successful elements of the experience. The design and story production was highly praised, and its characters and dramatic events engaged and immersed the audiences in the story world, bringing them closer to the protagonist's struggles rooting for her and her dreams. The audience wanted to know more about the heroine's future and lamented the open ended finale, or the lack of happy ending. The positive involvement and care for the protagonist of the fiction brought the audience closer to the island heritage, values and local culture. In resonance with [21, 52], the audience involvement with the main character was quite effective highlighting values and issues important for the local community at the time of the fiction, as well as at the present day, such issue being highlighted by the seven themes of the TS (Natural disasters, Laurisiva Medicinal Plants, Madeira's Fauna, Exotic Species, Hydrological Balance and Macaronesia Forests).

The FoL Hypermedia Platform component of the TS worked as a stimulus to further explore the island and its heritage, understand its local values and get closer to its community. Visitors felt like they were getting to know the locals and empathize with them. The visitors enjoyed the scientific content, as well as the "next-door neighbour" opinions, giving their insights and sometimes contrasting perspectives on island realit. Backed up by encouraging results from MTE and UEQ scales we can report that the combination of the two TS components worked in harmony reinforcing and integrating each other. The Hedonistic dimension, associated with pleasure, excitement and enjoyment, scored very highly for both components. Furthermore, the 'local culture' dimension, from the MTE scale, measuring the travellers' experience of friendly local people and 'meaningfulness' of the travel, indicates travellers' engagement in personally significant activities [10] was high.

These results indicate that we can answer RQ2 positively. The FoL TS was overall successful in invoking emotionally connection with the island local values and community and that the combination of the two TS components supported tourists to emotionally connect with the island local values and its community in a complementary but equal way. Results for our evaluation can confirm that Transmedia can be an effective strategy to foster empathy with a tourist destination's community, heritage and values.

Finally, concerning RQ3 (Did FoL TS enrich the visitors knowledge about the local destination cultural and natural heritage in a significant way? And if so, what role did the different TS components play out?), results from different measures report on encouraging outcomes. Tourists learned new facts and enriched their knowledge about several topics regarding the local destination heritage and culture. High values in the "Knowledge" MTE dimension and the Likert item "Transmedia Experience enabled learning about Madeira local values" support RQ3's first claim. Moreover, from the interviews' data, we recorded participants praising the nature of the information delivery, defining it as an "unusual blend between fiction and facts", effectively condensed and allowing visitors to get an engaging "first overview of Madeira's culture". Moreover, we were able to quantify how much participants enriched their knowledge. Results show significant differences in the pre and post TS questionnaires measuring the visitor's knowledge about the local destination.

In particular, answering the second part of RQ3, FOL LAMS helped participants in acquiring new knowledge on the destination (compared to before the the start of TS experience). After engaging with the FoL HP the knowledge increased once again. Participants acquired knowledge that was later reinforced and augmented after interacting with FoL HP, 
resulting in a cumulative effect. Looking at the participants MQS scores, the score increase was higher after the FoL HP and one could argue that FoL HP might have been most effective in enriching participants' knowledge. This is somewhat expected due to the factual and scientific nature of its content and the journalistic style of the delivery. Nevertheless, the FoL LAMS and in particular the fictional narrative generated empathy with nature preservation mission of its protagonist, generating care for the island natural patrimony and interests for the historical facts and even curiosity about the locations that played a role in Laura's story.

Finally we can answer positively RQ3 by reporting visitors enrichment of their knowledge about the island, after the TS experience, and also highlight the different roles played by the two components, regarding acquisition of factual information and empathy towards the community values and heritage.

\subsection{Limitations}

Despite the positive results stemming from our evaluations, we are aware of several limitations concerning our work.

Moyle et al. [45] call for importance of the inclusion of the visitors' perspectives in studies about interaction between locals and visitors in touristic islands. In trying to address this gap, several challenges were encountered in the strategies to reach tourists and engage them in the evaluation process. The research conducted could benefit from a larger sample of participants, though tourists are transient individuals only visiting destinations for a limited time. On reflection, some of the barriers were found when applying our methodology. The visitors are on holiday and/or on tourist mode with their time wisely planned, so participating in a research study is not a priority for them. Pressure to get through the study was often an issue and some of the experience results might have been affected by this. Another limitation of this research related to the difficulty of engaging tourists in evaluations is that we had to be quite flexible regarding the recruitment process and offer the experience to a broad tourism segment.

Moreover, visitors to the island have a variety of interests and preferred approaches to the local culture and nature, encouraging us to continue to collect and present the rich variety of stories about the cultural and natural heritage of the Island, highlighting the limitations in terms of content of the FoL TS experience. More information about the history of the Island, facts about its economy, and the impact of tourism on the archipelago, were all desired topics that could be further expanded in the TS experience.

Finally, we would like to highlight that further evaluations in different destinations' contexts would be needed in order to generalize the findings to other TS experiences, specially under different cultural characteristics, different concerns and local values.

\section{Conclusion and future work}

As digital technologies provide tools to empower audiences to participate in new interactive storytelling experiences, TS is spreading as a technique to engage, inspire and gather tourists around sensitive topics and move them closer to local destination values. In this article, we described the design and implementation of FoL, a bespoke Transmedia Storytelling experience, composed of two interconnected components: FoL LAMS and FoL HP. The extensive evaluation of the FoL TS reported promising results, answering the three research questions positively. It proved to be a successful example of a TS experience that contributes to engaging 
its audience while delivering local heritage information and fostering empathy and respect regarding local heritage and community. We hope that our work encourages the comparative design and evaluations of such TS experiences. However, evaluating TS experiences to enhance the tourist experience remains a challenge to overcome, tourists do not have much spontaneous time to spend following evaluation protocols. Future work should focus on the development of alternative analytical measures for the tourism experience that do not rely solely on self-reporting feedback and not interfere with the experience itself. Furthermore, it would be valuable to run a study that evaluates the impact of the TS experience, both on the visitors' experience and the local ecosystem in the long run.

Supplementary Information The online version contains supplementary material available at https://doi.org/ 10.1007/s1 1042-021-10949-2.

Acknowledgments We wish to acknowledge, the fellow researchers Claudia Silva, Dina Dionisio, Paulo Bala. Rui Trindade and Sandra Câmara for all their helpfull input and contribution towards the design of Fragments of Laura. We also wish to acknowledge all the participants of our study for their time and feedback.

Authors' contributions Both authors contributed for the design to the FoL TS experience. The first author was responsible for designing/conducting the FoL evaluation and data analysis. The second author as the principal investigator of the project supervised all the project stages and findings of this work. All authors discussed the results and contributed to the final manuscript.

Funding LARSyS (Projeto Estratégico LA 9 - UID/EEA/50009/2013); MITIExcell (M1420-01-0145- FEDER000002) Fundação Ciência e Tecnologia: PD/BD/114142/2015.

Data availability Not applicable.

Code availability Not applicable.

\section{Declarations}

Conflicts of interest/competing interests Not applicable'.

Open Access This article is licensed under a Creative Commons Attribution 4.0 International License, which permits use, sharing, adaptation, distribution and reproduction in any medium or format, as long as you give appropriate credit to the original author(s) and the source, provide a link to the Creative Commons licence, and indicate if changes were made. The images or other third party material in this article are included in the article's Creative Commons licence, unless indicated otherwise in a credit line to the material. If material is not included in the article's Creative Commons licence and your intended use is not permitted by statutory regulation or exceeds the permitted use, you will need to obtain permission directly from the copyright holder. To view a copy of this licence, visit http://creativecommons.org/licenses/by/4.0/.

\section{References}

1. Ask a Local app - Best recommendations from locals (2017) http://askalocalapp.com/. Accessed 21 Sep 2017

2. Avouris NM, Yiannoutsou N (2012) A review of Mobile location-based games for learning across physical and virtual spaces. J UCS 18:2120-2142 
3. Ballagas R, Kuntze A, Walz SP (2008) Gaming tourism: lessons from evaluating REXplorer, a pervasive game for tourists. In: Indulska J, Patterson DJ, Rodden T, Ott M (eds) Pervasive computing. Springer, Berlin Heidelberg, pp 244-261

4. Basso KH (1996) Wisdom sits in places: landscape and language among the Western apache, 1st edn. University of New Mexico Press, Albuquerque

5. Bell M, Chalmers M, Barkhuus L, et al (2006) Interweaving Mobile games with everyday life. In: Proceedings of the SIGCHI conference on human factors in computing systems. ACM, New York, NY, USA, pp. 417-426

6. Bilandzic M, Foth M, De Luca A (2008) CityFlocks: designing social navigation for urban Mobile information systems. In: Proceedings of the 7th ACM conference on designing interactive systems. ACM, New York, NY, USA, pp. 174-183

7. Braun V, Clarke V (2006) Using thematic analysis in psychology. Qual Res Psychol 3:77-101. https://doi. org/10.1191/1478088706qp063oa

8. Brombach G (n.d.) Death at Berlin Wall. https://sprylab.com/en/projekte/mobile-edutainment-app-deathberlin-wall

9. Center for Responsible Travel (2015) The Case for Responsible Travel: Trends \& Statistics 2015

10. Chandralal L, Valenzuela F-R (2015) Memorable tourism experiences: scale development. Contemp Manag Res 11:291-310. https://doi.org/10.7903/cmr.13822

11. Christou E, Sigala M (2002) Innovation in hospitality and tourism education. Int J Tour Res 4:65-67. https://doi.org/10.1002/jtr.330

12. Ciolfi L, McLoughlin M (2012) Designing for meaningful visitor engagement at a living history museum. In: proceedings of the 7th Nordic conference on human-computer interaction: making sense through design. ACM, pp 69-78

13. Cohen E (1979) A phenomenology of tourist experiences. Sociology 13:179-201. https://doi.org/10.1177/ 003803857901300203

14. Cool Cousin: City Guides Curated by Locals (2017) https://www.coolcousin.com/. Accessed 27 Sep 2017

15. Croes RR (2006) A paradigm shift to a new strategy for small island economies: embracing demand side economics for value enhancement and long-term economic stability. Tour Manag 27:453-465. https://doi. org/10.1016/j.tourman.2004.12.003

16. Davis JE (2012) Stories of change: narrative and social movements. SUNY Press

17. de Souza e Silva A (2009) Hybrid reality and location-based gaming: redefining mobility and game spaces in urban environments. Simul Gaming 40:404-424. https://doi.org/10.1177/1046878108314643

18. de Souza e Silva A, Delacruz GC (2006) Hybrid reality games reframed: potential uses in educational contexts. Games Cult 1:231-251. https://doi.org/10.1177/1555412006290443

19. Dionisio M, Nisi V, Nunes N, Bala P (2016) Transmedia storytelling for exposing natural capital and promoting ecotourism. In: Nack F, Gordon AS (eds) Interactive Storytelling. Springer International Publishing, pp. 351-362

20. Dionisio M, Silva C, Nisi V (2019) Fostering interaction between locals and visitors by designing a community-based tourism platform on a Touristic Island. In: Lamas D, Loizides F, Nacke L et al (eds) Human-computer interaction - INTERACT 2019. Springer International Publishing, Cham, pp 768-787

21. Ferreira SAM (2015) Location based Transmedia storytelling: enhancing the tourism experience. Universidade Lusófona

22. Ferreiraa S, Alvesa AP, Quicob C (2014) Location based Transmedia storytelling in social media-Peter's TravelPlot Porto case study. E rev tour res ERTR ENTER 2014Conference

23. Fleming L (2013) Expanding Learning Opportunities with Transmedia Practices: Inanimate Alice as an Exemplar J Media Lit Educ 8

24. Garcia A (2017) Transmedia: redefining where and how stories are told. J Adolesc Adult Lit 60:715-717. https://doi.org/10.1002/jaal.639

25. Goeldner CR (2006) Tourism: principles, practices, philosophies, 10th ed. J. Wiley, Hoboken, N.J

26. Gosling SD, Rentfrow PJ, Swann WB Jr (2003) A very brief measure of the big-five personality domains. J Res Personal 37:504-528. https://doi.org/10.1016/S0092-6566(03)00046-1

27. Green MC, Brock TC (2000) The role of transportation in the persuasiveness of public narratives. J Pers Soc Psychol 79:701-721. https://doi.org/10.1037/0022-3514.79.5.701

28. Green MC, Brock TC, Kaufman GF (2004) Understanding media enjoyment: the role of transportation into narrative worlds. Commun Theory 14:311-327. https://doi.org/10.1111/j.1468-2885.2004.tb00317.x

29. Hancox D (2017) From subject to collaborator: Transmedia storytelling and social research. Convergence 23:49-60. https://doi.org/10.1177/1354856516675252

30. Hardy A, Beeton RJS, Pearson L (2002) Sustainable tourism: an overview of the concept and its position in relation to Conceptualisations of tourism. J Sustain Tour 10:475-496. https://doi.org/10.1080/ 09669580208667183 
31. Helle S, Salmi H, Turunen M, et al (2017) MIRACLE handbook : guidelines for mixed reality applications for culture and learning experiences

32. Jaffe E (2019) The app that turns tourists into locals. In: CityLab http://www.theatlanticcities.com/ technology/2013/08/urban-buddy-turns-tourists-locals/6513/. Accessed 28 Jan 2019

33. Jenkins H (2008) Convergence culture: where old and new media collide, updated and with a new afterword. New York Univ. Press, New York, NY

34. Kim J-H (2014) The antecedents of memorable tourism experiences: the development of a scale to measure the destination attributes associated with memorable experiences. Tour Manag 44:34 45. https://doi.org/10. 1016/j.tourman.2014.02.007

35. Kim J-H, Ritchie JRB (2014) Cross-cultural validation of a memorable tourism experience scale (MTES). J Travel Res 53:323-335. https://doi.org/10.1177/0047287513496468

36. Kitto HDF (1968) Greek tragedy: a literary study. Methuen, London

37. Laurisilva of Madeira World Heritage Centre (2014). In: Periodic Report - Section II

38. Lee KM (2004) Presence, explicated. Commun Theory 14:27-50. https://doi.org/10.1111/j.1468-2885. 2004.tb00302.x

39. Loqal - Android Apps on Google Play (2017) https://play.google.com/store/apps/details?id=com.integral. loqal\&hl=en. Accessed 21 Sep 2017

40. MacCannell D (1976) The tourist: a new theory of the leisure class. University of California Press

41. Makarechi K (2012) "Bear 71": interactive film at Sundance tells dark side of human interaction with wildlife. In: bear 71 interact. Film Sundance tells dark side hum. Interact. Wildl. http://www.huffingtonpost. com/2012/01/23/bear-71-interactive-film-sundance n 1225040.html

42. Millard DE, Hargood C (2015) A research framework for engineering location-based poetics. ACM press, pp $13-16$

43. Miner's Walk (2019) http://minerswalk.com. Accessed 21 Jul 2019

44. Moyer-Gusé E (2008) Toward a theory of entertainment persuasion: explaining the persuasive effects of entertainment-education messages. Commun Theory 18:407-425. https://doi.org/10.1111/j.1468-2885. 2008.00328.x

45. Moyle B, Weiler B, Croy G (2010) Tourism interaction on islands: the community and visitor social exchange. Int J Cult Tour Hosp Res 4:96-107. https://doi.org/10.1108/17506181011045172

46. Murphy ST, Frank LB, Moran MB, Patnoe-Woodley P (2011) Involved, transported, or emotional? Exploring the determinants of change in knowledge, attitudes, and behavior in entertainment-education. J Commun 61:407-431. https://doi.org/10.1111/j.1460-2466.2011.01554.x

47. Neuhofer BE (2014) An exploration of the technology enhanced tourist experience. Bournemouth University

48. Nisi V, Haahr M (n.d.) Weird View: Interactive Multilinear Narratives and Real-Life Community Stories. Crossings 4:13

49. Nisi V, Oakley I, Haahr M (2006) Inner City locative media: design and experience of a location-aware Mobile narrative for the Dublin liberties neighborhood. In: Intelligent Agent

50. Nisi V, Oakley I, Posthuma De Boer M (2010) Locative narratives as experience: a new perspective on location aware multimedia stories. Porto, Portugal

51. Nisi V, Dionísio M, Silva C, Nunes NJ (2019) A participatory platform supporting awareness and empathy building between tourists and locals: the Há-Vita case study. In: Proceedings of the 13th biannual conference of the Italian SIGCHI chapter: designing the next interaction. Association for Computing Machinery, Padova, pp 1-10

52. Nobrega R, Jacob J, Coelho A, et al (2017) Mobile location-based augmented reality applications for urban tourism storytelling. In: 2017 24 Encontro Português de Computação Gráfica e Interação (EPCGI). IEEE, Guimaraes, pp. 1-8

53. O'Brien HL, Cairns P, Hall M (2018) A practical approach to measuring user engagement with the refined user engagement scale (UES) and new UES short form. Int J Hum-Comput Stud 112:28-39. https://doi.org/ 10.1016/j.ijhcs.2018.01.004

54. Packer HS, Hargood C, Howard Y, Papadopoulos P, Millard DE (2017) Developing a Writer's toolkit for interactive locative storytelling. In: Nunes N, Oakley I, Nisi V (eds) Interactive storytelling. Springer International Publishing, Cham, pp 63-74

55. Pearce PL, Moscardo GM (1986) The concept of authenticity in tourist experiences. Aust N Z J Sociol 22: 121-132. https://doi.org/10.1177/144078338602200107

56. Pigram JJ, Wahab S (1997) Tourism, development and growth. Taylor \& Francis Ltd., Hoboken

57. Pine BJ, Gilmore JH (2011) The experience economy. Harvard Business Press

58. Pittarello F (2011) Designing a context-aware architecture for emotionally engaging Mobile storytelling. In: Proceedings of the 13th IFIP TC 13 international conference on human-computer interaction - volume part I. Springer-Verlag, Berlin, pp 144-151 
59. Pratten R (2012) The Roswell experience - transmedia storytelling for America's small towns

60. Pratten R (2020) Transmedia for Change. http://www.tstoryteller.com/transmedia-for-change. Accessed 20 Jan 2020

61. Quiring T (n.d.) Sustainable Stories: Integrated Transmedia as an Ecology of Storymaking

62. Salvat B, Pailhe C (2002) Islands and coral reefs, population and culture, economy and tourism : world view and a case study of French Polynesia documentation Ifrecor. Tourism, biodiversity and information 14(2002):213-231

63. Schank RC, Abelson RP (1995) Knowledge and memory: the real story. In: Wyer RS (ed) knowledge and memory: the real story. Lawrence Erlbaum associates, pp 1-85

64. Scheyvens R, Momsen J (2008) Tourism in Small Island states: from vulnerability to strengths. J Sustain Tour 16:491-510. https://doi.org/10.1080/09669580802159586

65. Silva C, Bettencourt A, Dionísio M, et al (2017) Há-Vita: a transmedia platform about Madeira's nature and culture. In: 2017 sustainable internet and ICT for sustainability (SustainIT). Pp 1-2

66. Slater MD, Rouner D (2002) Entertainment - education and elaboration likelihood: understanding the processing of narrative persuasion. Commun Theory 12:173-191. https://doi.org/10.1111/j.1468-2885. 2002.tb00265.x

67. Stivers C (1993) Reflections on the role of personal narrative in social science. Signs J Women Cult Soc 18: 408-425. https://doi.org/10.1086/494800

68. Stray Boots - scavenger hunt (n.d.) https://www.strayboots.com/

69. Things to Do in - Well Anywhere / Your Personal Tour Guide | Vayable (2017) https://www.vayable.com/. Accessed 27 Sep 2017

70. Tussyadiah IP (2014) Toward a Theoretical Foundation for experience design in tourism. J Travel Res 53: 543-564. https://doi.org/10.1177/0047287513513172

71. User Experience Questionnaire (UEQ) (2019) https://www.ueq-online.org/. Accessed 3 Jun 2019

72. Wang N (1999) Rethinking authenticity in tourism experience. Ann Tour Res 26:349-370. https://doi.org/ 10.1016/S0160-7383(98)00103-0

73. Wang D, Park S, Fesenmaier DR (2012) The role of smartphones in mediating the touristic experience. J Travel Res 51:371-387. https://doi.org/10.1177/0047287511426341

74. Weber J (n.d.) Augmented Reality Gaming: A new Paradigm for Tourist Experiences? 11

75. Welcome to Pine Point (2017) In: IDFA DocLab. https://www.doclab.org/2010/welcome-to-pine-point/. Accessed 20 Sep 2017

76. World Travel and Tourism Council (2017) World Travel and Tourism Council: Economic Impact 2017

77. Wu B, Wang AI (2011) A pervasive game to know your city better. IEEE, pp:117-120

78. Xu F, Buhalis D, Weber J (2017) Serious games and the gamification of tourism. Tour Manag 60:244-256. https://doi.org/10.1016/j.tourman.2016.11.020

79. Zheng Y, Capra L, Wolfson O, Yang H (2014) Urban computing: concepts, methodologies, and applications. ACM Trans Intell Syst Technol 5:1-55. https://doi.org/10.1145/2629592

80. Zimmerman J, Forlizzi J, Evenson S (2007) Research through design as a method for interaction design research in HCI. In: Proceedings of the SIGCHI conference on Human factors in computing systems. ACM, pp. 493-502

Publisher's note Springer Nature remains neutral with regard to jurisdictional claims in published maps and institutional affiliations. 